\title{
Sostenibilidad de la Agroindustria Nacional en términos de recursos naturales
}

Sustainability of the National Agro-Industry in terms of natural

resources

Recibido: abril 15 de 2019 | Revisado: junio 06 de 2019 | Aceptado: julio 10 de 2019

\begin{abstract}
Alexis Dueñas ${ }^{1,2}$ Resumen
Julián CCASAni ${ }^{1}$ El problema de la contribución del capital natural en las trayectorias de Maribel Huatuco ${ }^{\mathrm{I}, 3}$ crecimiento de la agroindustria regional cobra actualidad y representa un estancamiento del crecimiento de la economía nacional basada en actividades

VAnessa García ${ }^{\mathrm{I}}$ extractivas, en la cual la agroindustria, juega un rol importante en la reactivación

DANIEL INGA ${ }^{\mathrm{I}}$ del crecimiento económico del país. El objetivo de este trabajo es determinar y establecer la influencia del capital natural en las trayectorias de crecimiento de la agroindustria, en términos de producción, en el Perú. Los resultados obtenidos, en cuanto a tasas de crecimiento, señalan que para la agroindustria de tipo C3 tiene patrones mixtos, similar al caso de las cadenas del tipo C2 y C1. En cuanto, a presiones y cargas ambientales se halló una de presión del 15 al 25\% para el suelo, del 25 y $40 \%$ para el agua, con una generación de residuos sólidos ( 45 y 60\%) para las cadenas del tipo $\mathrm{C} 1$. Se determinó que entre el 25 y $35 \%$ son las presiones en el suelo, y que el 40 a $45 \%$ de tales volúmenes descansan en las demandas de agua. Por otro lado, el 28 al $35 \%$ de los volúmenes producidos se traducen en residuos para las cadenas $\mathrm{C} 2$, tendencia que es extensible a las cadenas C3. Se concluye que la agroindustria nacional sigue por el modelo expansivo, es decir, a mayor crecimiento mayor consumo de capital natural (recursos naturales) y mayor generación de contaminantes.
\end{abstract}

Palabras clave: Agroindustria, capital natural, sostenibilidad, crecimiento y recursos naturales

\begin{abstract}
The problem of the contribution of natural capital in the regional agro-industrial growth trajectories charges today as result of the stagnation of the growth of the national economy based on extractive activities as the agriculture, would play an important role in the revival of the country's economic growth. In that context, the central objective of the research is to "determine and establish the influence of natural capital in growth trajectories of agro-industry, in terms of production, the Peru. The results, in terms of growth rates, indicate that for the agroindustry of type $\mathrm{C} 3$ has mixed patterns, similar to the case of $\mathrm{C} 2$ and $\mathrm{C} 1$ type chains. As to pressures and environmental loads found a pressure of 15 to $25 \%$ for flooring, $25 \%$ and $40 \%$ for water, with a generation of solid waste ( 45 to $60 \%$ ) for chains of type $\mathrm{C} 1$. It was determined that you between 25 and $35 \%$ are pressures on the ground, and that 40 to $45 \%$ of such volumes are part of the demands for water. On the other hand, 28 to $35 \%$ of the produced volumes translate into waste for $\mathrm{C} 2$, trend that is extensible to the $\mathrm{C} 3$ chains. National agribusiness continues
\end{abstract} 1 Universidad Nacional by the expansive model, namely growing greater consumption of natural capital

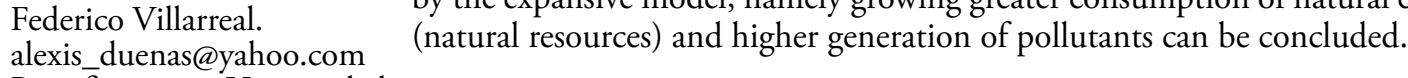

2 Pontificia Universidad Católica del Perú.

Key words: Agribusiness, natural capital, sustainability, growth and natural

3 Universidad Le CondonBleu resources

(C) Los autores. Este artículo es publicado por la Revista Campus de la Facultad de Ingeniería y Arquitectura de la Universidad de San Martín de Porres. Este artículo se distribuye en los términos de la Licencia Creative Commons Atribución No-comercial - Compartir-Igual 4.0 Internacional (https://creativecommons.org/licenses/ CC-BY), que permite el uso no comercial, distribución y reproducción en cualquier medio siempre que la obra original sea debidamente citada. Para uso comercial contactar a: revistacampus@usmp.pe.

https://doi.org/10.24265/campus.2019.v24n28.04 


\section{Introducción}

Elproblemadelacontribucióndelcapital natural en las trayectorias de crecimiento de la agroindustria regional cobra actualidad como consecuencia del estancamiento del crecimiento de la economía nacional basa en actividades extractivas, y en la cual, las agro-exportaciones y en particular la agroindustria, jugaría un rol importante en la reactivación de la senda del crecimiento económico del país. En el 2000 las agroexportaciones bordeaban 500 millones de dólares. En el 2015, registraron la cifra de 5400 millones de dólares (Prom Perú, 2015). En el plano teórico, desde la economía clásica hasta la reciente economía ecológica, se preguntan si el nivel de vida alcanzado por las naciones se mantendrá. Esta reflexión teórica no es unidimensional, porque no se refiere únicamente al crecimiento económico, entendido como el aumento cuantitativo de la riqueza, de los bienes y servicios que tienen los territorios, léase de la agroindustria nacional. Sino se refiere también a los cambios cualitativos que tienen relación con la calidad de vida, finalmente con el bienestar y el desarrollo que tal crecimiento genera en la sociedad.

El desarrollo sostenible ha sido abordado desde dos tradiciones teóricas, donde, por ejemplo, una de ellas la define como proceso económico que afecta directamente el bienestar humano. La sostenibilidad se expresa económicamente como la utilidad per cápita que no decae a lo largo del tiempo. La otra tradición considera los recursos que existen en la sociedad para generar bienestar o consumo, a partir del concepto de capital (Hanley \& Atkinson, 2003). Por tanto, el capital puede ser visto como un sistema de tres grupos de variables: ecológicas, económicas y socio-culturales (Chiesura \& de Groot, 2003). Schumacher hizo la primera referencia sobre el capital natural (Schumacher, 1973), al señalar que la economía y la satisfacción de las necesidades humanas, dependen del medio ambiente. Esta idea estuvo asociada, en un inicio a los recursos naturales no renovables, como el petróleo. Hoy en día se hace difícil suponer cualquier desarrollo económico, al margen de los factores ambientales, sin ellos su perdurabilidad en el largo plazo está en duda(SIEE, 1992). El antecedente más preciso le corresponde a John Mill, quién postuló el modelo de "economía de estado estable", según la cual, las economías desarrolladas tienden a estabilizar su ritmo de crecimiento "material"(Costanza \& et.al., 1999).

En tiempos más recientes, el concepto de capital natural se ha difundido gracias a los trabajos de Pearce, que propone considerar la relación entre economía y sustentabilidad (Pearce \& Turner, 1995). El "capital natural" al igual que los recursos naturales está impregnado de la visión antropocéntrica, en cuanto a su valor monetario(Schumacher, 1973). En el otro lado, se tienen a los defensores del "equilibrio total del capital", que como el Banco Mundial (2005). Desde la otra vertiente, se propone incluir los servicios ambientales en el capital natural, a partir de los ciclos bióticos y de materiales, como las funciones de absorción y dilución de contaminantes, así como un flujo constante de energía que recibe el planeta en forma global (Wackernagel \& Rees, 1997).

Históricamente, la economía clásica identificó tres tipos de capital: tierra, trabajo y capital, entendido como financiero y frecuentemente denominado como "capital". El ulterior desarrollo 
del pensamiento económico omitió en la representación de las funciones de producción a la tierra, con excepción de la economía campesina, y centrando su atención en el trabajo y el capital. Posteriormente, y dada la creciente preocupación sobre el papel de los recursos naturales en la producción, algunas funciones de la producción incluyeron a la energía y los materiales (Ekins, Simon, Deutsch, \& Folke, 2003). El hombre ha convertido el "capital natural" en una gama amplia de sistemas artificiales como: agricultura, acuicultura, silvicultura, entre otros, Ello ha sido posible porque los ecosistemas naturales presentan varias características ambientales que a su vez determinan su capacidad de proporcionar bienes y servicios, por ejemplo, aire, agua, tierra y hábitat. Esto atributos brindan flujos ambientales que satisfacen las necesidades humanas (De Groot., 1992).

El criterio de sostenibilidad afirma que no todo el "capital natural" puede sustituirse, y que existe una porción denominada "crítico" que no puede sustituirse por el capital humano o hecho, y debe conservarse individualmente (Chiesura A. d., 2003). La sostenibilidad fuerte considera que la sustitución del capital natural está limitado por características ambientales tales como: irreversibilidad, incertidumbre y existencia de los componentes "criticos" del cual depende la contribución al bienestar social (Ekins, Simon, Deutsch, \& Folke, 2003).

En el pensamiento económico subsisten, desde hace algún tiempo, dos concepciones sobre el desarrollo. Ambas posiciones han dado lugar a la formulación de dos modelos: exógeno y endógeno, cuyas derivaciones prácticas se resumen en el siguiente apartado. En la literatura, este debate también ocurre como consecuencia de la forma como se ha explicado la relación entre el crecimiento y el ambiente. Una primera perspectiva ha sido la óptica micro-económica que conlleva a considerar las externalidades que emergen de la producción y que se expresan como contaminación y degradación del medio. Sin embargo, el agotamiento de los recursos como consecuencia de la expansión de la producción y del consumo conlleva a una óptica diferente, de escala macro (Schumpeter, 1934). El crecimiento se basa en la creación de empresas y en ella la tecnología juega un papel fundamental (Shumpeter, 1982). Para Dasgupta, la escasez de materias primas no es un problema para el crecimiento económico, porque puede ocurrir una sustitución de tales recursos (Dasgupta, 1993).

Una pregunta en esta dirección es saber ¿si la sustitución es posible? Esta sería viable si se cumplen los siguientes supuestos: el ambiente se cuidará por las preferencias de los agentes económicos que buscan la calidad ambiental, si hay suficiente sustitución entre el flujo de recursos y el stock de capital. Si el modelo a considerar es una función de producción lineal y suponen que la producción aumenta el stock de contaminación, generándose externalidades negativas (Michel \& Rotillon, 1992). De otro lado, si en la función de producción considera al ambiente como un recurso renovable, entonces los aspectos ambientales reducen el crecimiento, a largo plazo (Bovenberg \& Smulders, 1995). Ahora si los recursos no son renovables, aquellos que se vayan a utilizar para alcanzar el crecimiento serán a costa de comprometer el stock de las futuras generaciones. Por tanto, los posibles escenarios serían: Desplazar la inversión hacia el capital natural, si se postula la "sustitución", entonces habría que aplicar una política que maximice su 
productividad actual e incrementar su oferta futura y llevar a cabo una política fiscal, con incentivos para las empresas a emplear cualquier medio para incrementar su producción afectando negativamente al entorno ambiental.

\section{Método}

\section{Caracterización de las unidades de análisis}

Del 2000 al 2010, el sector de la agroindustria creció a tasas muy importantes, y ello se refleja, según el volumen de las exportaciones, Figura 1 (Arteaga, s/f). Si se analiza el volumen de las exportaciones no tradicionales, se podrá observa que las agro-exportaciones, que incluyen a las exportaciones agrarias y agroindustriales, indicador de crecimiento, representan en promedio el $60 \%$ de las exportaciones no tradicionales. En la década siguiente el panorama es menos auspicioso y la velocidad del crecimiento se detendrá para algunos componentes.

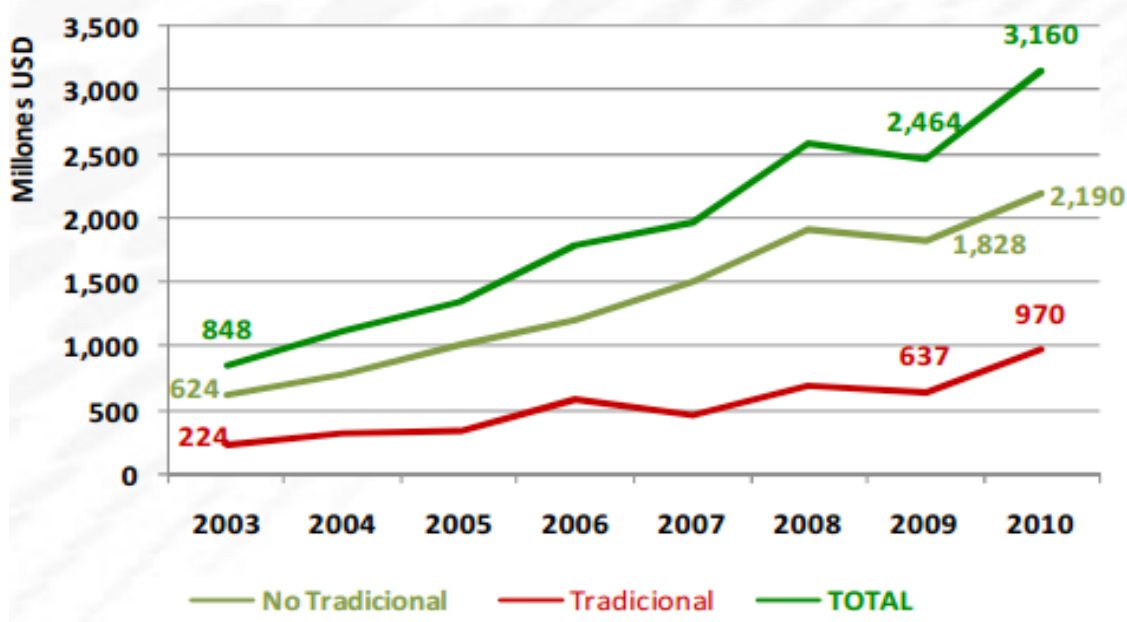

Figura 1. Evolución de las exportaciones peruanas (2003-2010) Fuente: (Arteaga, s/f)

Una forma alternativa a la anterior, consiste en evaluar la dinámica del sector a la luz de los volúmenes producidos. Es decir, hacer una evaluación por medio de un indicador "intra sectorial" como es el volumen producido. Se aprecian importantes dispersiones entre los diferentes sectores. Por su varianza y desviación estándar, las mayores heterogeneidades se dan en la producción de aceite, grasas, avena, entre otras. La menor heterogeneidad se da en derivados de cacao, sémola, y derivados lácteos. En cuando a la simetría de las distribuciones se tiene que la producción de alimento balanceado se aproximaría a una distribución simétrica $(\mathrm{SK} \approx 0)$ al darse un valor de 0.17. Las distribuciones de aceite, grasas, avenas, derivados lácteos, arroz pilado, fideos y harina son del tipo asimétricas de cola derecha $(\mathrm{SK}>0)$. En cambio, las distribuciones de datos de derivados de cacao, sémola, leche $\mathrm{y}$ embutidos y carnes son asimétricas de cola izquierda $(\mathrm{SK}<0)$. Por último, en cuanto a la agudeza del apuntamiento se tiene que la producción de sémola correspondería a una curva mesocúrtica $(\mathrm{K}=0)$. Las demás distribuciones presentan curvas del tipo platicurticas $(\mathrm{K}<0)$. 


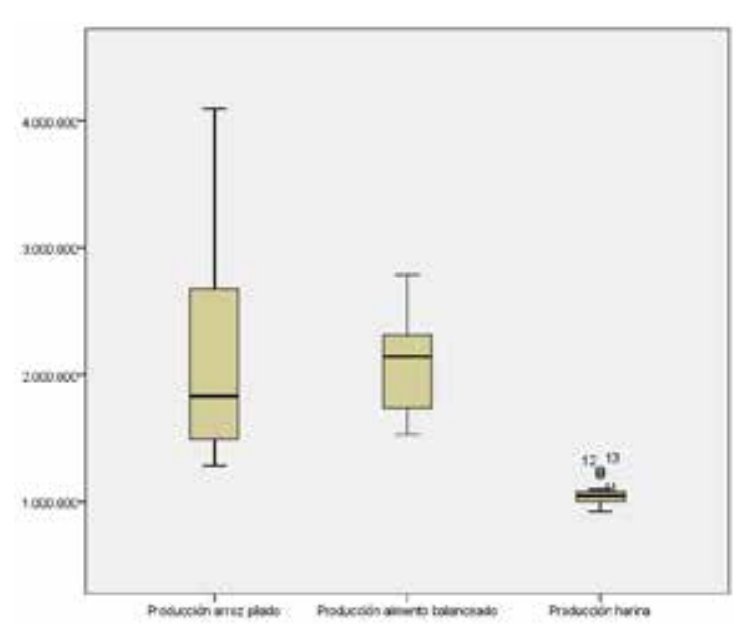

Figura 2-A: Diagramas de caja para arroz pilado, alimento balanceado y harina

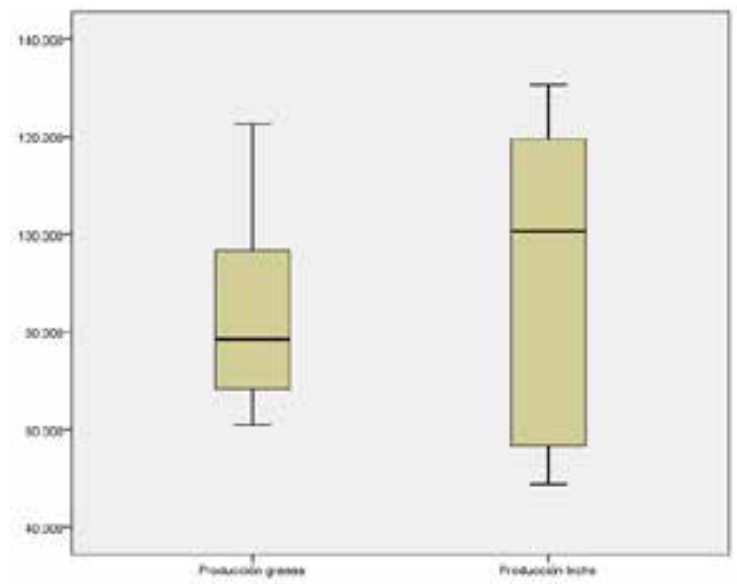

Figura 2-C: Diagramas de caja para grasas y leche

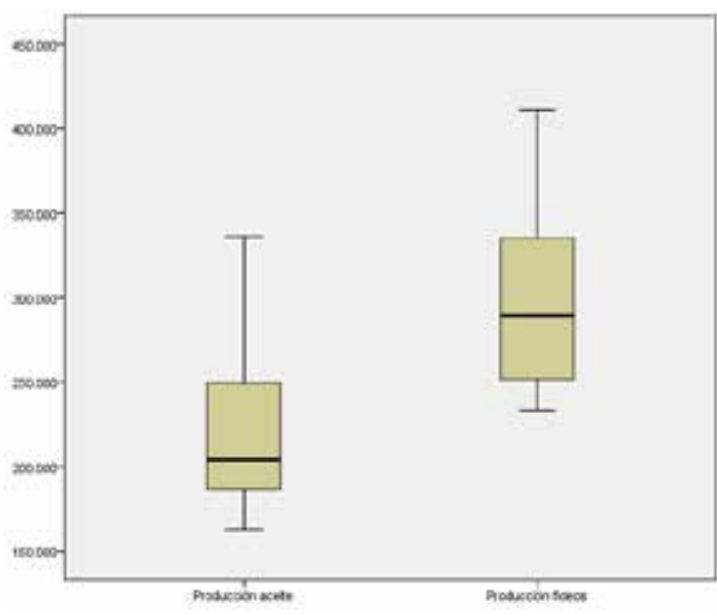

Figura 2-B: Diagramas de caja para harina $\mathrm{y}$ fideos

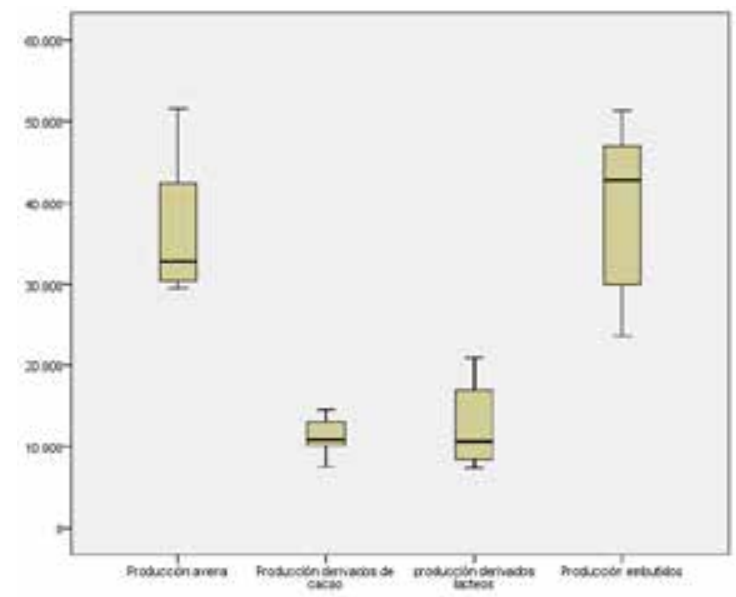

Figura 2-D: Diagramas de caja para avena, derivados de cacao, derivados lácteos y embutidos y carnes

Figura 2. Diagramas de caja para los componentes subsectoriales de la agroindustria. Periodo 2000-2016

Fuente: Base de datos INEI

Los diagramas de cajas ilustran, precisamente, los patrones heterogéneos descritos en el anteriormente. Las mayores dispersiones se aprecian en la producción de arroz pilado (Fig. 2-A), producción de fideos (Fig. 2-B), producción de leche (2-C) y avena y embutidos (Fig. 2-D). Estas heterogeneidades deben tratarse adecuadamente en la descomposición de las series de tiempo que se describen en el apartado de resultados.

\section{Procedimiento de cálculo, estimación de índices y tasas}

Para el caso de la hipótesis principal y derivadas se asume que el capital natural influye en las trayectorias de crecimiento de la agroindustria del país. Esto significa que el crecimiento es visto como variable dependiente del capital natural que dispone el sector, en este caso, agroindustria del país. 
El crecimiento sectorial debe ser visto como una variación de la producción función del tiempo, el cual puede ser expresado como una tasa, con la formulación siguiente (Chiang \& Wainwrigth, 2006):

$$
T_{c}=\frac{\operatorname{Pro}_{2}-\operatorname{Pro}_{1}}{\operatorname{Pro}_{1}}
$$

El problema con la expresión de la tasa de crecimiento es la naturaleza de su trayectoria, en este caso, la tasa debería ajustarse a un modelo exponencial o polinómico, si se añadiese, en este último caso, una restricción de aumento, y todo ello en función del tiempo. De esa forma, la tasa podría convertirse en una razón de cambio en función del tiempo, y la cual además depende del tamaño de los recursos involucrados, y cuya expresión estaría dada por (Gujarati, 2006):

$$
\theta_{t}=\frac{\partial P B I}{\partial T}\left[q_{t}\right]
$$

En el caso de las estimaciones de los recursos naturales empleados o inputs, así como de los residuos sólidos resultantes (output) se estimaron sobre la base de diagramas de flujo estandarizados, cuyos valores estandarizados se aprecian en el Apéndice I.

\section{Procedimiento de análisis}

Para esto debe tener en cuenta que los datos a trabajarse constituyen variaciones temporales de la producción, y obviamente del capital natural. Una vez elegidos las series de tiempo, debe considerarse el análisis de cada una de ellas, con la técnica de descomposición, de forma tal, que sea posible identificar y "aislar" los componentes de corto, mediano y largo plazo, sin que implique alteración de las frecuencias de las series originarias, controlando en todo momento que no se generen ciclos artificiales o espurios (Escobal, 2002).

Una técnica usada es el método de la descomposición, por el cual se obtienen tres aspectos importantes; uno relacionado con la tendencia $\left(b_{t}\right)$, el segundo, estacional referido a la presencia de ciclos repetitivos $\left(S_{t}\right)$, y finalmente el componente estacionario $\left(\mathrm{I}_{t}\right)$ referido a las características estocásticas del comportamiento de las variables en el tiempo. Debe también tomarse en cuenta la naturaleza de la varianza de la serie. Si la varianza resultará ser constante, entonces deberían aplicarse el "modelo aditivo" y si esta fuese no constante sería mejor emplear el "modelo multiplicativo"(Veliz, 2009):

$$
\begin{gathered}
Y_{t}=b_{t}+S_{t}+I_{t} \\
Y_{t}=\left(a+b_{t}\right) * S_{t} * I_{t}
\end{gathered}
$$

Normalmente, la regresión es el método más empleado para ver el comportamiento dela variación temporal. Es posible que el nivel de variación de la producción [Pro] respecto al $\mathrm{K}_{\mathrm{n}}$ pudiera verse influenciado por la disponibilidad de activos ambientales provenientes de periodos anteriores, sobre todo en el caso de recursos no renovables, o de algunos renovables como la biomasa, agua o suelo. Esto supondría que tal vez no exista una relación "no contemporánea" o retardada entre $\mathrm{Y}$ o Pro y $\mathrm{X}$ o $\mathrm{K}_{\mathrm{n}}$. Así se tendría $\mathrm{Y}_{1}=$ al Pro en el tiempo t, $X_{1}=K_{n}$ en el tiempo $\mathrm{t}{ }_{\mathrm{X}-1}=\mathrm{al} \mathrm{K}_{\mathrm{n}}$ en el momento $(\mathrm{t}-1), \mathrm{y} \mathrm{X}_{\mathrm{t}-}$ ${ }_{2}=\mathrm{K}_{\mathrm{n}}$ en el momento ( $\left.\mathrm{t}-2\right)$, por tanto, se configura el modelo siguiente (Gujarati, 2006):

$$
Y_{t}=A+\beta_{0} X_{t}+\beta_{1} X_{t-1}+\beta_{2} X_{t-2}+u_{t}
$$


También es posible generalizar la expresión anterior, obteniendo un modelo sencillo de retardos distribuidos con $\mathrm{k}$ periodos, bajo la expresión de (Gujarati, 2006; Veliz, 2009):

$Y_{t}=A+\beta_{0} X_{t}+\beta_{1} X_{t-1}+\beta_{2} X_{t-2}+\ldots+\beta_{k} X_{t-k}+u_{t}(6)$

Este modelo expresa que el efecto de las variaciones unitarias sobre el valor de la variable explicativa se percibe en $\mathrm{k}$ periodos. En este contexto, la expresión es el multiplicador de corto plazo o de impacto (Gujarati, 2006). De otro lado,

$$
\begin{aligned}
& \text { Correlaciones positivas } \\
& \text { Si } \mathrm{e}_{\mathrm{jt}}>0 \mathrm{y} \mathrm{e}_{\mathrm{it-k}}>0 \rightarrow \text { puntaje 1; } \\
& \text { Si } \mathrm{e}_{\mathrm{jt}}<0 \mathrm{y} \mathrm{e}_{\mathrm{jt-k}}<0 \rightarrow \text { puntaje 1; } \\
& \text { Si } \mathrm{e}_{\mathrm{jt}}>0 \mathrm{y} \mathrm{e}_{\mathrm{it}-\mathrm{k}}<0 \rightarrow \text { puntaje } 0 \text {; } \\
& \mathrm{Si}_{\mathrm{jt}}<0 \mathrm{y} \mathrm{e}_{\mathrm{it-k}}>0 \rightarrow \text { puntaje } 0 \text {. }
\end{aligned}
$$

Por tanto, siguiendo lo descrito por Escobal es posible generar un puntaje $\theta$, con la expresión siguiente, que constituiría el índice de sostenibilidad sectorial (Escobal, 2002):

$$
\theta_{t}=\frac{\sum_{t=0}^{T=N} \text { Puntaje }_{i t}}{N-1}
$$

\section{Resultados}

Los resultados que se exponen tienen una naturaleza exploratoria. Se pasa revista a series de tiempo que ilustran la evolución de la producción de este sector en el periodo comprendido entre 2000-2016, para ello se ofrece un análisis estilizado de tales curvas, que tiene el objeto determinar las tasas de crecimiento o decrecimiento para el escenario antes referido. Se evaluó además los eslabonamientos internos de cada uno de los subsectores, esto es agregando los subsectores y no por ramas de actividad, siguiendo la clasificación la variación media de $(\mathrm{Y})$ en los siguientes periodos, expresada por $y$, cuyas sumas parciales se denominan multiplicadores intermedios. Con esta técnica es posible distinguir el corto, mediano y largo plazo.

Un siguiente paso consiste en obtener los puntajes de tendencia, bajo las consideraciones expuesta $\theta$ y sus correspondientes puntajes de giro, o los $\theta^{\prime}$ sugeridos antes. En el primer caso, el criterio de puntaje de tendencia se obtiene si se cumple (Escobal, 2002):

\section{Correlaciones negativas}

Si $\mathrm{e}_{\mathrm{jt}}>0 \mathrm{y} \mathrm{e}_{\mathrm{it-k}}<0 \rightarrow$ puntaje 1;

Si $\mathrm{e}_{\mathrm{jt}}<0 \mathrm{y} \mathrm{e}_{\mathrm{jt- \textrm {k }}}>0 \rightarrow$ puntaje 1 ;

Si $\mathrm{e}_{\mathrm{jt}}>0$ y e $_{\mathrm{it}-\mathrm{k}}>0 \rightarrow$ puntaje 0 ;

Si $\mathrm{e}_{\mathrm{jt}}<0 \mathrm{y} \mathrm{e}_{\mathrm{it}-\mathrm{k}}<0 \rightarrow$ puntaje 0 .

del CIIU, por el contrario se agrupan los sectores siguiendo la complejidad de la cadena del valor propuesta por Porter \& Heppelmann (2016), de ese modo se distinguen las agroindustrias que pertenecen a las cadenas $(\mathrm{C} 1)$ o de baja transformación, en las cuales los productos solo se seleccionan, limpian, empacan y almacenan con o sin cadena de frío. Luego están las agroindustrias de cadenas (C2) de mediana transformación, que suponen la obtención de productos mediante procesos de transformación física de sus materias primas $y$, por último, están las agroindustrias del tipo (C3), que implican la transformación tanto física como química de sus materias primas.

\section{Análisis comparativo del crecimiento según agro-cadenas}

Nuestro análisis se basa en agrupar a los diferentes subsectores en función del grado de transformación de sus 
productos, siguiendo la teoría de Michael Porter (2015), de esa forma se tiene que en la cadena de tipo $\mathrm{C} 1$ existen dos subsectores que representan el $18.1 \%$ de los casos analizados. En la cadena de tipo C2 Tabla 1 se tiene al $45.5 \%$ de los casos y finalmente en la cadena de tipo C3 se tienen al $36.4 \%$ de los subsectores analizados y comprendidos en este primer reporte de investigación (Tabla 1).

Distribución de los sectores según nivel de transformación

\begin{tabular}{llll}
\hline \multicolumn{1}{c}{ Cadenas } & \multicolumn{1}{c}{$\begin{array}{c}\text { Cadenas tipo C1 } \\
\text { (Transformación básica) }\end{array}$} & $\begin{array}{c}\text { Cadenas C2 } \\
\text { (Transformación física) }\end{array}$ & $\begin{array}{c}\text { Cadenas C3 } \\
\text { (Transformación química) }\end{array}$ \\
\hline & Arroz pilado & Alimento balanceado & Aceites \\
Leche & Fideos & Grasas \\
Sub sectores & & Harina & Derivados lácteos \\
& & Sémola & Embutidos y carnes \\
& & Avena & \\
Numero de cadenas & 2 & Derivados de cacao & \\
Porcentaje & $18.1 \%$ & 5 & $46.4 \%$ \\
\hline
\end{tabular}

La evolución de las tasas de crecimiento de cada uno de los sectores en función de las cadenas de valor, que se agruparon según las consdieraciones descritas para la Tabla 1, señalan, que para las agroindustria de tipo C3 se observan patrones mixtos.
Siendo más aguda las depresiones en el caso de los derivados lácteos (2003 y 2013). Menores crisis presentan, en su crecimiento, los subsectores aceites y embutidos (Figura 3).

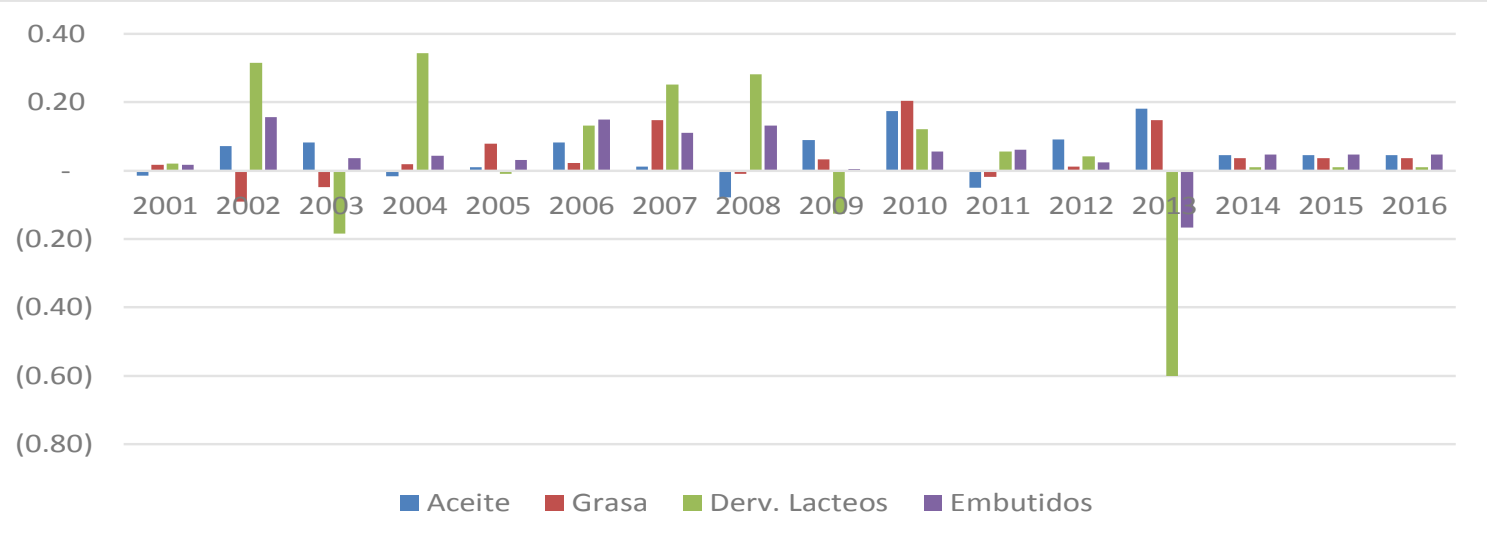

Figura 3. Tasas de crecimiento de los sectores de cadena productiva tipo C3

Un caso aparte constituye el tema del crecimiento del subsector alimento balanceado, que por sus importantes volúmenes de producción y complejas tasas de crecimiento debe ser evaluado de forma separada de los demás subsectores que integran las cadenas de tipo C2. Asíse tiene que el sector atraviesa un dinamismo volátil para 2000-2005 (Figura 4), con una notoria tendencia a la baja en sus velocidades de crecimiento, seguidas de un periodo breve de recuperación (20062007) y otra vez se observa un valle prolongado entre 2008-2010, con una relativa recuperación en 2011 para luego volver a bajar entre 2014 y 2016. Nótese el peor retroceso se produce en el 2013. 


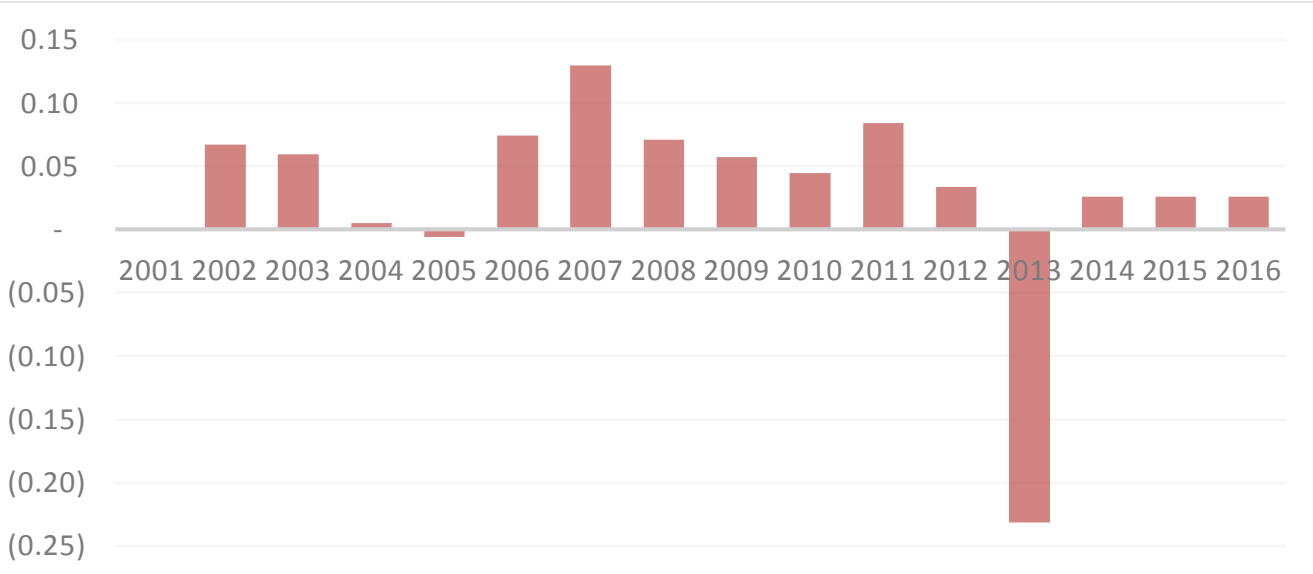

Figura 4. Tasas de crecimiento de los sectores de cadena productiva tipo C2, el caso de la producción de alimento balanceado.

Respecto a los otros componentes de esta cadena $\mathrm{C} 2$, se tiene que el subsector de derivados de cacao es mucho más volátil que el resto de componentes, con tasas crecientes y decrecientes de relativa importancia. Por ejemplo, en los años 2003 y 2004, presentó tasas de 46 y 48\%, y después de 2011 con tasas de crecientes del orden del 12 y $20 \%$ al año siguiente (2013). Los demás subsectores tienen un comportamiento más estilizado y cuyas variaciones son menos volátiles que las anteriores (Figura 5).

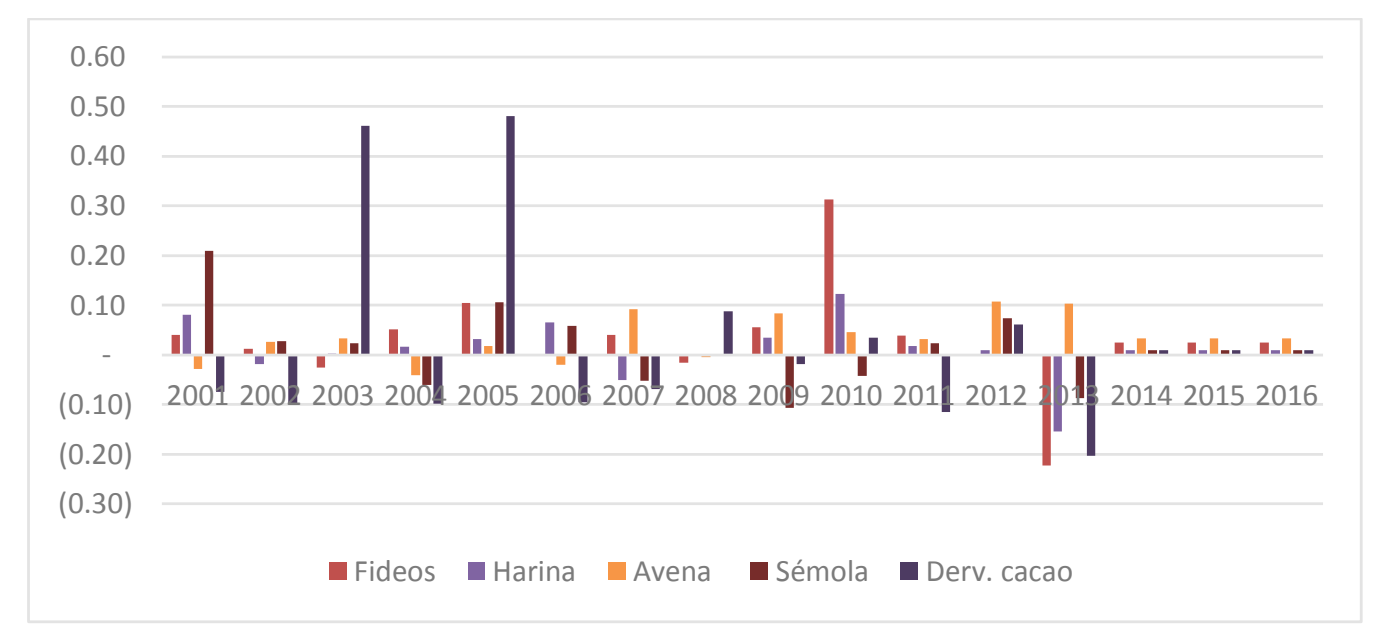

Figura 5. Tasas de crecimiento de los sectores de cadena productiva tipo C2, excepto alimento balanceado.

El análisis correspondiente para el caso de los subsectores que pertenecen a la cadena C1, (Figura 6), según la cual se aprecia que tanto la producción de leche como de arroz pilado, tienen una importante volatilidad en el crecimiento. En el primer caso, se tienen tasas crecientes muy importantes de 21 y $48 \%$ en los años 2007 y 2008 , respectivamente, pero a la vez se observan tasas decrecientes muy acentuadas 12 y $19 \%$ en 2006 y 2013, respectivamente. Por otro lado, en el caso del arroz las tasas crecientes más importantes son $34 \%$ en $2005,46 \%$ en 2012 y $24 \%$ en 2013 . Y las tasas negativas se presentan en el año 2004 (-13\%), 2006 $(-5 \%)$ y $2011(-7 \%)$. 


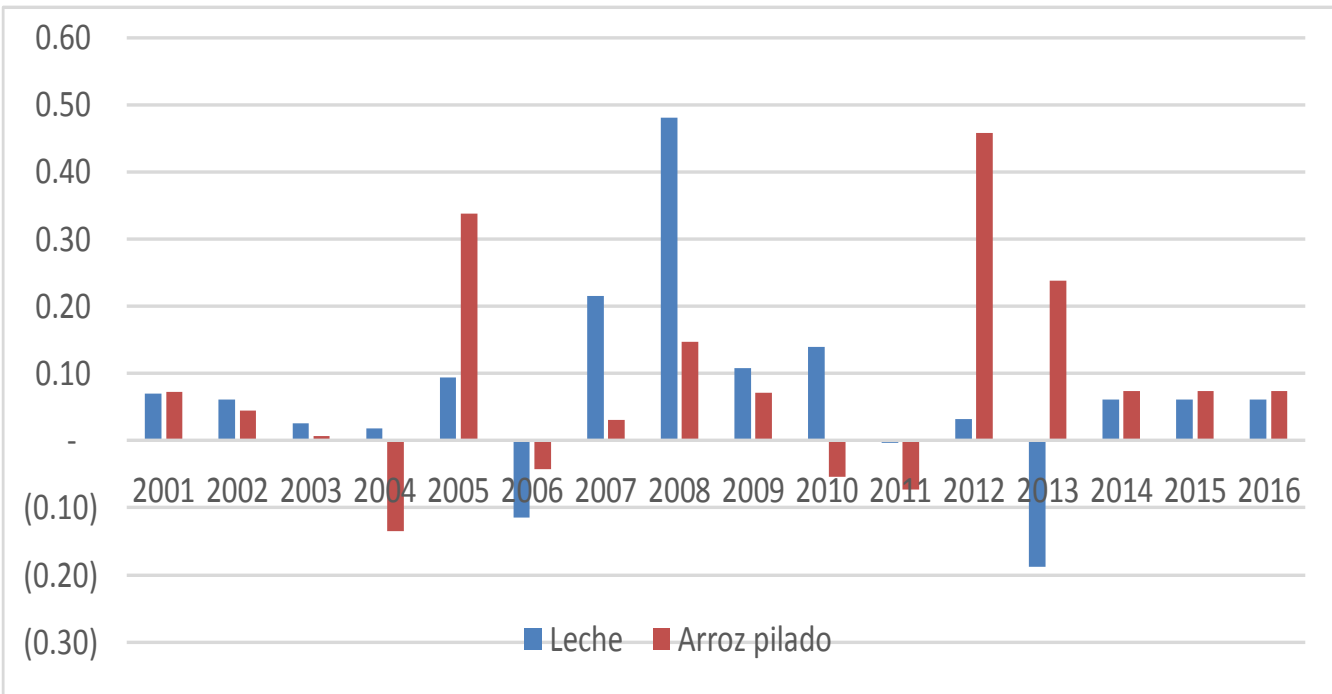

Figura 6. Tasas de crecimiento de los sectores de cadena productiva tipo C1.

\section{Una aproximación al uso del capital natural en la agroindustria nacional}

Diversas fuentes señalan que aún en los sistemas económicos más avanzados y eficientes del mundo (Escot \& Galindo, 1999; Chiesura \& de Groot, 2003; Ekins, Simon, Deutsch, \& Folke, 2003), una proporción del crecimiento depende del capital natural, léase recursos naturales. Si bien es cierto, que en ese

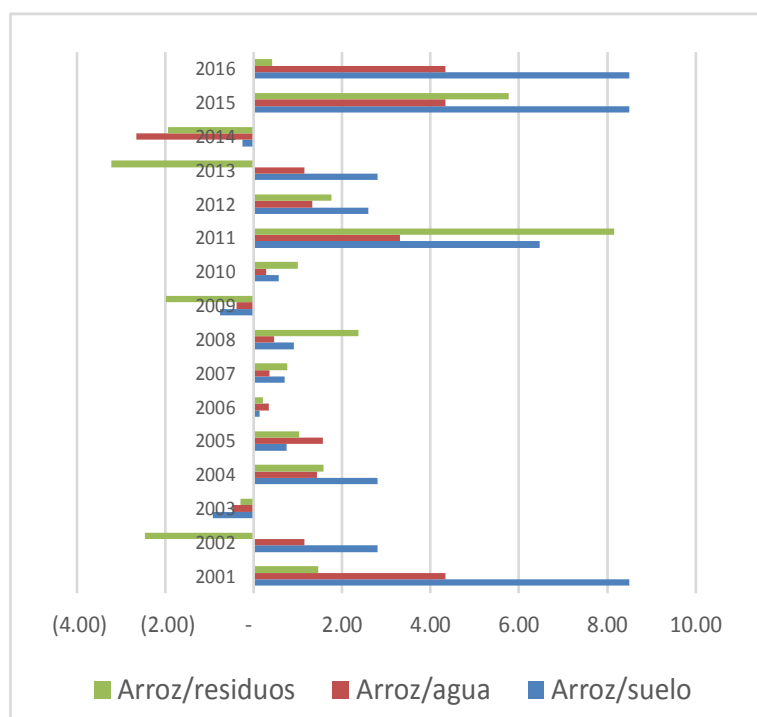

punto de la discusión los expertos se dividen en dos grandes grupos. De un lado, están aquellos que afirman que el crecimiento de las economías no depende sustancialmente de los recursos naturales, y además postulan una relación adversa, a la que se ha denominado la "maldición de los recursos naturales". Y de otro, están los que afirman que los recursos naturales explican el crecimiento de las economías, en particular en la fase de expansión.

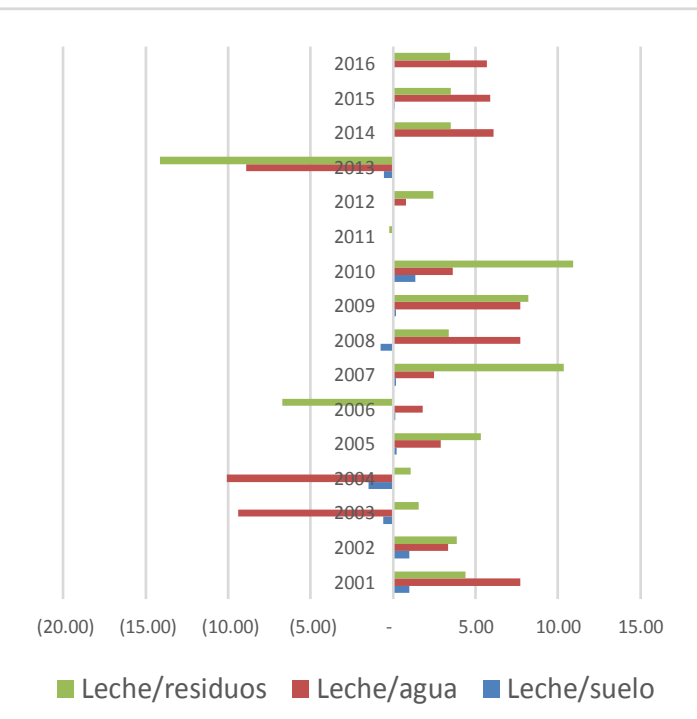

Figura 7. Tasas de consumo de recursos naturales y generación de residuos en C1. Fig. 7-A: Tasas de consumo de recursos naturales (suelo \& agua) y de generación de residuos para $\mathrm{C} 1$ (caso arroz pilado). Fig. 7-B: Tasas de consumo de recursos naturales (suelo \& agua) y de generación de residuos para C1 (caso leche pasteurizada). 
En la Figura 7 se aprecian tasas diferencias tanto a nivel de cargas como de presiones ambientales, entendidas las primeras como descargas de contaminantes, en este caso de residuos sólidos, y las segundas expresadas como tasas de consumo de recursos naturales (suelo y agua) para las cadenas agroindustriales del tipo $\mathrm{C} 1$. También se estima que existen notables diferencias respecto al tipo de productos, bien sea leche pasteurizada o arroz pilado.

Las tasas de cambio, bien sea para recursos naturales (suelo y agua) como para los contaminantes (residuos sólidos) difieran además por periodo temporales. Así se tienen tasas intensivas, muy alta para recursos naturales en los años de mayor expansión de la producción y viceversa. De esta forma que constataría que la relación entre consumo de capital natural y crecimiento productivo, al menos para los casos estudiados de las cadenas productivas del tipo C1 para el periodo 2000-2016. Una evidencia adicional se ofrece en la Tabla 2, donde se observa que el aumento de la producción va aparejado de mayores volúmenes de consumo de agua e incrementos sustancias en la superficie. En este último, el lector podría preguntarse cuál es la relación entre productos agroindustriales como leche pasteurizada o arroz pilado con el consumo de suelo. La relación se produce como consecuencia que tanto la leche como el arroz dependen de extensas áreas para cultivar pastos y de ese modo alimentar a una población de vacas, las que luego proporcionarán volúmenes importantes de leche fresca. El caso del arroz pilado la relación es mucho más directa, porque se trata de asegurar una superficie de cultivo de arroz que permita venderse en los mercados regionales, y para ello se requiere, del servicio de pilado.

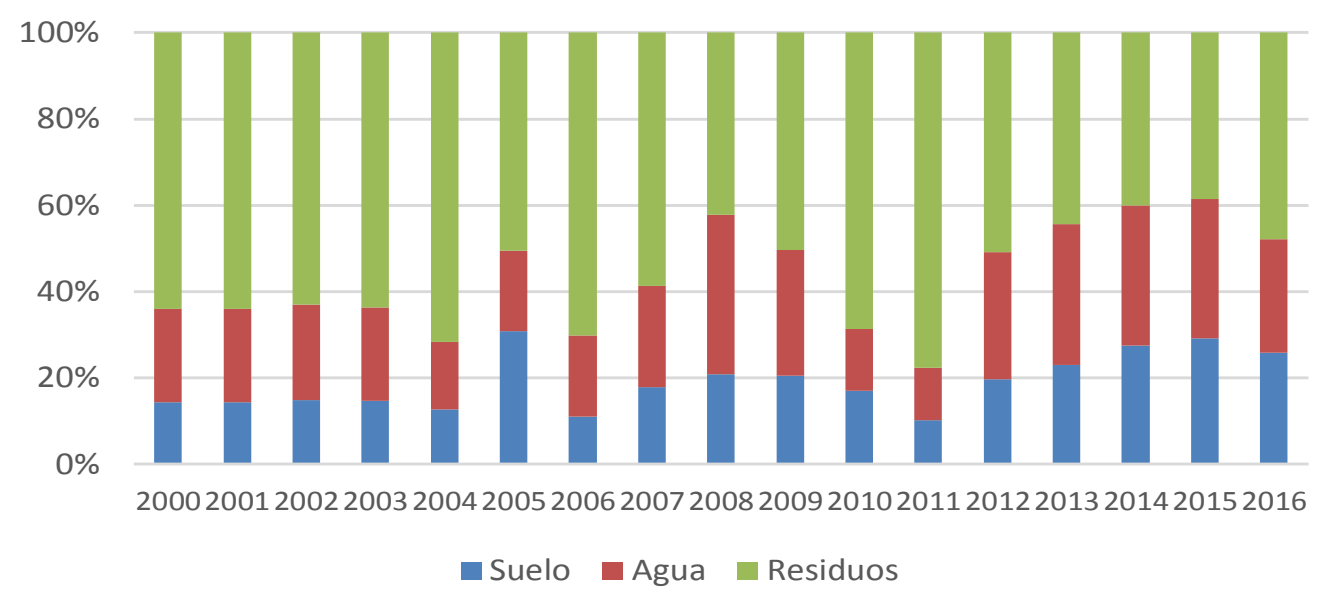

Figura 8. Composición de presiones y cargas ambientales en las cadenas agroindustriales de tipo $\mathrm{C} 1$

En la Figura 8 se observa, en términos proporcionales, para los volúmenes producidos y descritos en la Tabla 3, la proporción de uso de recursos naturales y generación de cargas ambientales en términos de residuos sólidos. Se aprecia un uso promedio una presión del 15 al $25 \%$ para el suelo, seguido del $25 \mathrm{y}$
$40 \%$ para el agua, con una proporción importante de generación de residuos sólidos, que oscila entre 45 y 60\%. Esto último indicaría que las cadenas agroindustriales del tipo $\mathrm{C} 1$ presentan importantes presiones ambientales pero muy significativas cargas, traducidas en volúmenes de residuos sólidos. 
Tabla 2

Producción, consumo de recursos naturales (capital natural) y generación de residuos para cadenas agroindustriales del tipo C1 (2000-2016)

\begin{tabular}{lcccccccc}
\hline & \multicolumn{2}{c}{ Producción total anual en tm } & \multicolumn{3}{c}{ Arroz pilado } & \multicolumn{3}{c}{ Leche } \\
Ańos & Arroz pilado & Leche & $\begin{array}{c}\text { Suelo } \\
\text { ha* }^{*}\end{array}$ & $\begin{array}{c}\text { Agua } \\
\mathbf{m}^{3 *}\end{array}$ & $\begin{array}{c}\text { Residuos } \\
\text { tm* }\end{array}$ & $\begin{array}{c}\text { Suelo } \\
\text { ha* }^{*}\end{array}$ & $\begin{array}{c}\text { Agua } \\
\text { m }^{3 *}\end{array}$ & $\begin{array}{c}\text { Residuos } \\
\text { tm}^{*}\end{array}$ \\
\hline 2000 & $1,324,471$ & 48,747 & $155,820.16$ & $305,509.90$ & $903,717.89$ & $48,720.81$ & $3,096.87$ & 11,149 \\
2001 & $1,420,103$ & 52,138 & $167,070.99$ & $327,568.91$ & $968,969.87$ & $52,109.98$ & $3,312.30$ & 11,925 \\
2002 & $1,482,619$ & 55,347 & $169,841.17$ & $333,000.29$ & $943,501.38$ & $55,346.49$ & $3,518.02$ & 12,754 \\
2003 & $1,492,684$ & 56,739 & $159,014.97$ & $311,773.81$ & $909,852.51$ & $53,014.88$ & $3,369.81$ & 13,641 \\
2004 & $1,291,427$ & 57,770 & $87,303.72$ & $171,172.66$ & $781,871.09$ & $52,975.21$ & $3,367.29$ & 14,590 \\
2005 & $1,727,850$ & 63,194 & $668,042.26$ & $448,894.27$ & $1,209,118.70$ & $78,465.34$ & $5,248.29$ & 15,605 \\
2006 & $1,654,448$ & 55,924 & $120,089.88$ & $235,455.06$ & $865,326.50$ & $18,487.45$ & $1,175.13$ & 16,691 \\
2007 & $1,704,593$ & 67,929 & $192,042.33$ & $376,529.14$ & $931,108.94$ & $94,943.07$ & $6,034.92$ & 17,852 \\
2008 & $1,955,786$ & 100,579 & $467,980.47$ & $917,549.19$ & $1,036,891.68$ & $52,757.65$ & $6,638.41$ & 19,094 \\
2009 & $2,093,810$ & 11,486 & $287,850.02$ & $564,375.17$ & $967,494.99$ & $114,881.11$ & $7,302.25$ & 20,422 \\
2010 & $1,981,961$ & 126,982 & $89,992.02$ & $176,443.49$ & $855,442.68$ & $126,369.22$ & $8,032.48$ & 21,843 \\
2011 & $1,837,121$ & 126,616 & $67,622.40$ & $132,584.32$ & $837,674.56$ & $44,836.82$ & $2,849.99$ & 23,362 \\
2012 & $2,678,374$ & 130,591 & $392,381.68$ & $769,325.90$ & $1,317,548.15$ & $124,827.12$ & $7,934.46$ & 24,987 \\
2013 & $3,316,726$ & 106,023 & $423,772.22$ & $830,871.97$ & $1,119,106.04$ & $168,197.43$ & $10,691.23$ & 26,725 \\
2014 & $3,559,396$ & 112,553 & $418,752.49$ & $821,030.00$ & $994,089.68$ & $283,200.75$ & $11,760.35$ & 28,585 \\
2015 & $3,819,821$ & 119,486 & $449,390.71$ & $881,101.06$ & $1,039,275.58$ & $358,032.49$ & $12,936.39$ & 30,573 \\
\hline 2016 & $4,099,300$ & 126,845 & $482,270.60$ & $945,567.24$ & $1,717,063.99$ & $462,119.17$ & $14,230.03$ & 32,700 \\
\hline
\end{tabular}

*Valores estimados con base a balances de masa por producto según diagramas de flujo de procesos estandarizados por sub-sector. Fuente: (Prom Perú, 2015) y estimaciones propias.

En el caso de las cadenas agroindustria del tipo C2, el comportamiento de la tasa varía sustancialmente, esto tiene sentido, porque el nivel de procesamiento y transformación en estas agroindustrias es mayor, dado que se trata de una alteración física de los productos agrarios, pecuarios y/o forestales, tal como se aprecia en la Figura 9. Por ejemplo, para el caso del alimento balanceado, donde además debe considerarse el efecto de importación derivado de los insumos maíz amarillo y soya. Un panorama similar se aprecia en los productos derivados del cacao y en menor intensidad en la producción de sémola. Con un comportamiento cualitativamente diferente se presenta en el caso de los productos del sector harinero, por ejemplo, fideos, harina y avena. En todos ellos, aprecian importantes tasas de consumo de recursos naturales, lo cual resulta lógico si se considera que, en la industria harinera, el nivel de relación es directo respecto a la superficie cultivada de trigo o avena. Aquí nuevamente debe considerarse el efecto de la importación, básicamente del trigo, que como se conoce, el Perú es un país deficitario en la producción de este cereal, no solo por consideraciones tecnológicas sino por restricciones de índole ecológica (Tejada, 2008). Argumento que también se extiende al caso de la avena, que, al formar parte de los cereales emparentados con el trigo, tienen las mismas restricciones ecológicas y tecnológicas para un cultivo más extendido y con mayor rendimiento. 


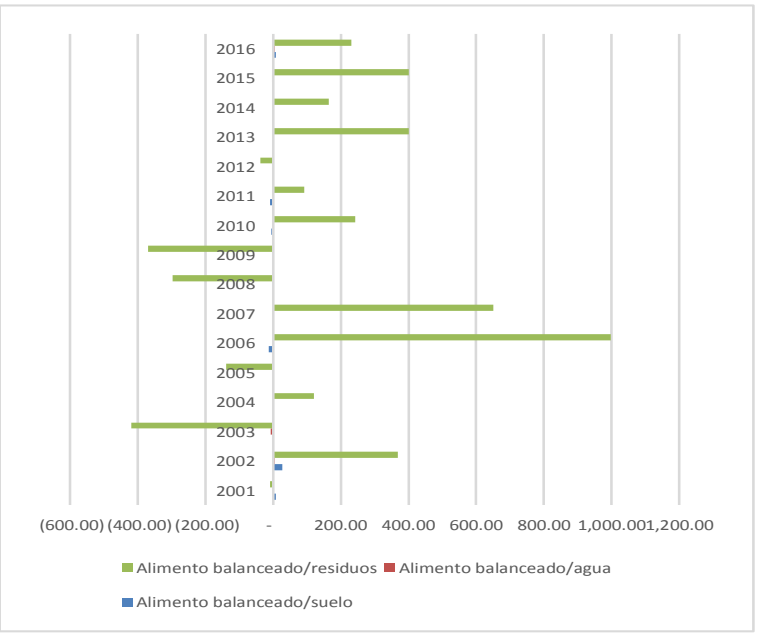

Fig. 9-A: Tasas de consumo de recursos naturales (suelo \& agua) y de generación de residuos para C2 (caso alimento balanceado).

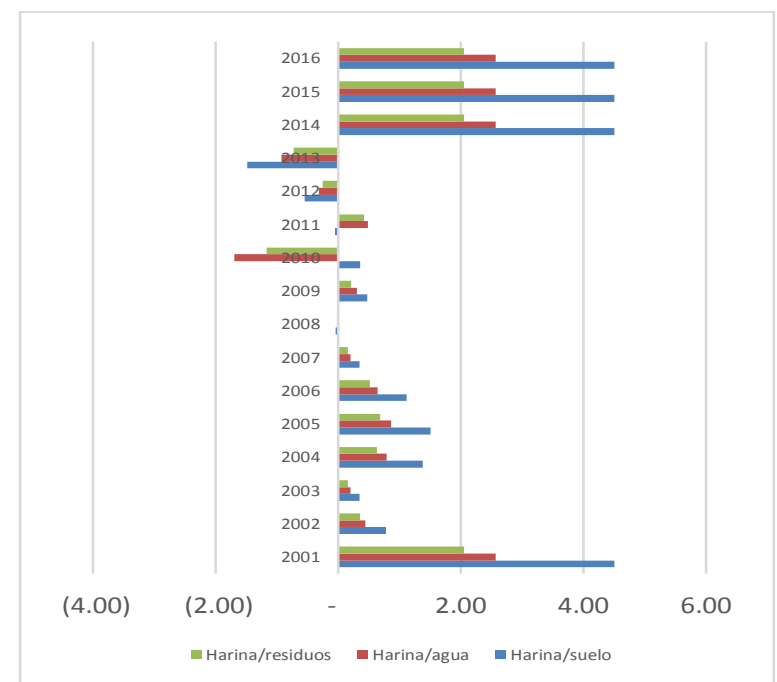

Fig. 9-C: Tasas de consumo de recursos naturales (suelo \& agua) y de generación de residuos para C2 (caso harina).

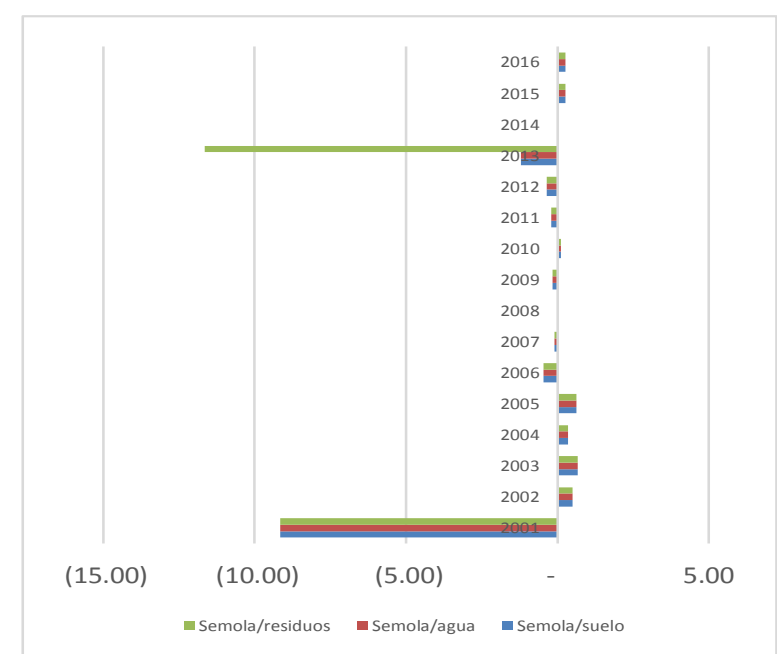

Fig. 9-E: Tasas de consumo de recursos naturales (suelo \& agua) y de generación de residuos para C2 (caso sémola).

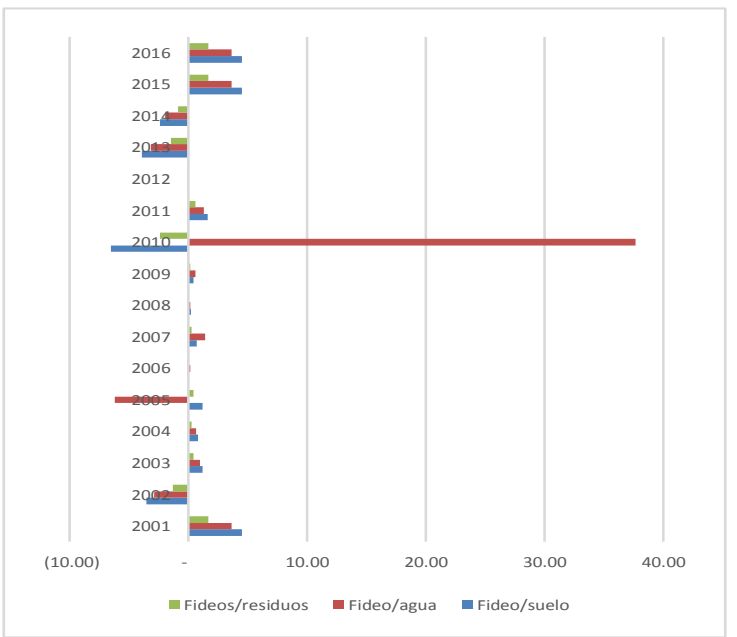

Fig. 9-B: Tasas de consumo de recursos naturales (suelo \& agua) y de generación de residuos para C2 (caso fideos).

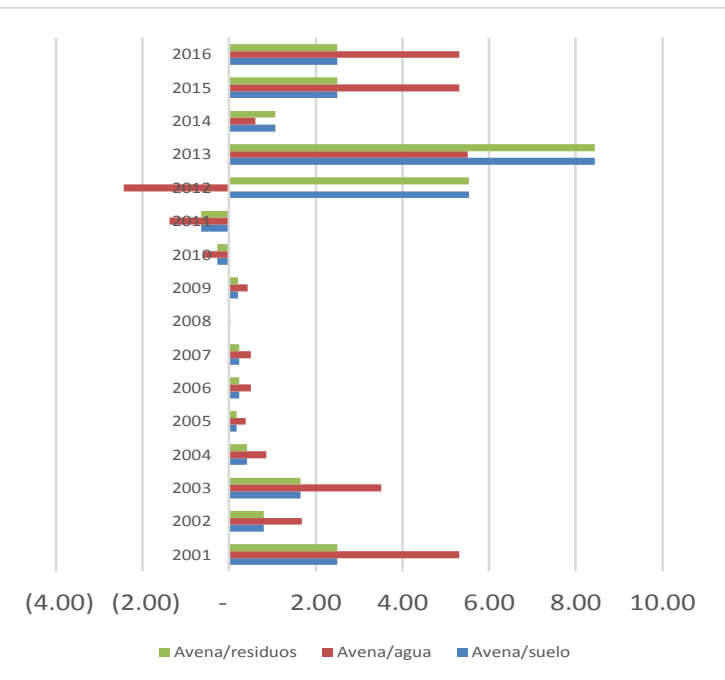

Fig. 9-D: Tasas de consumo de recursos naturales (suelo \& agua) y de generación de residuos para C2 (caso avena).

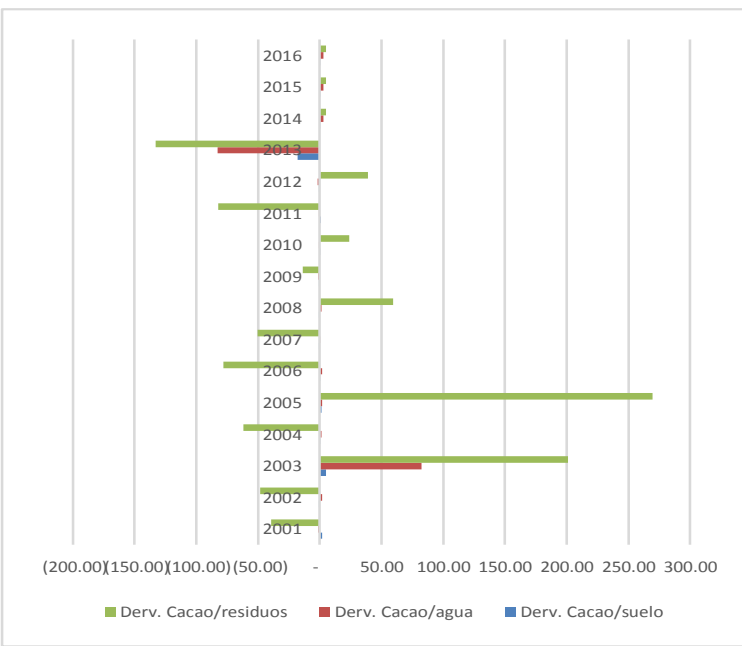

Fig. 9-F: Tasas de consumo de recursos naturales (suelo \& agua) y de generación de residuos para $\mathrm{C} 2$ (caso derivados de cacao).

Figura 9. Tasas de consumo de recursos naturales y generación de residuos en C2 
En la Tabla 3 se presentan tanto los volúmenes productos como el nivel de presión ambiental para las agroindustrias de tipo C2. Por las características de los productos analizados, en este caso se aprecia una mejor relación entre el volumen producido y los consumos de suelo y garua, así como de la generación de residuos sólidos. Nuevamente, se observa una trayectoria expansiva, según la cual a mayor producción mayores niveles de presión de recursos naturales y mayores cargas ambientales traducidas en residuos sólidos generados por estas cadenas.

Tabla 3

Producción, consumo de recursos naturales (capital natural) y generación de residuos para cadenas agroindustriales del tipo C2 (2000-2016)

\begin{tabular}{|c|c|c|c|c|c|c|c|c|c|c|c|c|c|c|c|}
\hline \multirow[b]{2}{*}{ Años } & \multicolumn{6}{|c|}{ Producción en tm } & \multicolumn{3}{|c|}{ Alimento balanceado } & \multicolumn{3}{|c|}{ Fideos } & \multicolumn{3}{|c|}{ Harina } \\
\hline & $\begin{array}{c}\text { Alimento } \\
\text { balanceado }\end{array}$ & Fideos & Harina & Avena & Sémola & $\begin{array}{l}\text { Derv. } \\
\text { cacao }\end{array}$ & $\begin{array}{l}\text { Suelo } \\
\text { ha* }^{*}\end{array}$ & $\begin{array}{l}\text { Agua } \\
\mathrm{m}^{3 *}\end{array}$ & $\begin{array}{l}\text { Residuos } \\
\mathrm{tm}^{*}\end{array}$ & $\begin{array}{l}\text { Suelo } \\
\text { ha* }^{*}\end{array}$ & $\begin{array}{c}\text { Agua } \\
\mathrm{m}^{3 *}\end{array}$ & $\begin{array}{c}\text { Residuos } \\
\mathrm{tm}^{*}\end{array}$ & $\begin{array}{l}\text { Suelo } \\
\text { ha* }^{*}\end{array}$ & $\begin{array}{l}\text { Agua } \\
\mathrm{m}^{3 *}\end{array}$ & $\begin{array}{l}\text { Residuos } \\
\text { tm }^{*}\end{array}$ \\
\hline 2000 & $1,536,976$ & 232,935 & 927,094 & 30,388 & 6,957 & 8,911 & 180,821 & 354,527 & 1,049 & 51,763 & 63,889 & 139,860 & 206,021 & 361,678 & 452,956 \\
\hline 2001 & $1,536,724$ & 242,466 & $1,001,941$ & 29,543 & 8,414 & 8,255 & 180,791 & 364,880 & 1,079 & 53,881 & 66,504 & 145,582 & 222,654 & 390,877 & 489,524 \\
\hline 2002 & $1,640,190$ & 245,445 & 983,146 & 30,334 & 8,646 & 7,443 & 184,455 & 384,133 & 1,225 & 53,043 & 65,469 & 143,318 & 198,364 & 348,236 & 436,122 \\
\hline 2003 & $1,738,012$ & 239,211 & 986,469 & 31,369 & 8,846 & 10,875 & 219,858 & 367,756 & 991 & 47,918 & 59,144 & 129,471 & 208,117 & 365,357 & 457,564 \\
\hline 2004 & $1,746,738$ & 251,698 & $1,002,638$ & 30,093 & 8,311 & 0808 & 227,973 & 319,858 & 1,063 & 63,448 & 78,311 & 171,430 & 219,864 & 385,980 & 483,391 \\
\hline 2005 & $1,736,443$ & 277,925 & $1,034,732$ & 30,630 & 9,188 & 14,528 & 185,406 & 457,239 & 1,138 & 84,969 & 74,065 & 229,579 & 241,174 & 423,390 & 530,242 \\
\hline 2006 & $1,865,639$ & 278,285 & $1,102,855$ & 30,045 & 9,724 & 13,148 & 174,520 & 642,594 & 1,267 & 52,166 & 76,287 & 140,949 & 302,033 & 530,231 & 664,048 \\
\hline 2007 & $2,107,259$ & 289,537 & $1,047,468$ & 32,813 & 9,215 & 12,240 & 298,826 & 357,751 & 1,639 & 68,317 & 84,321 & 184,586 & 143,637 & 252,161 & 315,800 \\
\hline 2008 & $2,256,849$ & 285,085 & $1,045,190$ & 32,655 & 9,212 & 13,324 & 479,038 & 486,824 & 1,136 & 45,728 & 56,440 & 123,552 & 202,464 & 356,208 & 445,136 \\
\hline 2009 & 2,385,633 & 300,855 & $1,081,105$ & 35,401 & 8,226 & 13,075 & 335,205 & 308,372 & 1,028 & 84,037 & 83,361 & 227,061 & 279,232 & 473,918 & 613,917 \\
\hline 2010 & $2,492,797$ & 395,034 & $1,214,252$ & 37,043 & 7,883 & 13,525 & 314,963 & 607,412 & 1,470 & 69,566 & 85,862 & 187,960 & 658,142 & 395,562 & 500,345 \\
\hline 2011 & $2,702,550$ & 410,564 & $1,236,460$ & 38,245 & 8,070 & 11,969 & 293,200 & 490,749 & 3,763 & 79,110 & 97,642 & 213,748 & 251,475 & 441,474 & 552,891 \\
\hline 2012 & $2,793,763$ & 411,095 & $1,248,038$ & 42,346 & 8,669 & 12,712 & 476,749 & 563,126 & 1,304 & 52,572 & 64,888 & 142,046 & 230,402 & 404,480 & 506,560 \\
\hline 2013 & $2,146,742$ & 319,492 & $1,055,119$ & 46,733 & 7,918 & 10,142 & 278,391 & 360,535 & 1,194 & 76,016 & 93,824 & 205,389 & 234,471 & 411,623 & 515,506 \\
\hline 2014 & $2,202,634$ & 327,353 & $1,065,671$ & 48,306 & 7,997 & 10,243 & 190,044 & 536,254 & 1,205 & 72,745 & 89,786 & 196,550 & 236,816 & 415,739 & 520,661 \\
\hline 2015 & $2,259,981$ & 335,407 & $1,076,327$ & 49,932 & 8,077 & 10,345 & 265,880 & 510,490 & 1,218 & 74,535 & 91,995 & 201,386 & 239,184 & 419,897 & 525,868 \\
\hline 2016 & $2,318,821$ & 343,659 & $1,087,091$ & 51,613 & 8,158 & 10,449 & 272,803 & 523,049 & 1,230 & 76,369 & 94,259 & 206,341 & 241,576 & 424,096 & 531,126 \\
\hline
\end{tabular}

* Valores estimados con base a balances de masa por producto según diagramas de flujo de procesos estandarizados por sub-sector.

Fuente: (Prom Perú, 2015) y estimaciones propias.

La tendencia descrita en el párrafo anterior se refuerza con los resultados ofrecidos en la Figura 10. De ese modo, se tiene que en promedio entre el 25 y $35 \%$ de los volúmenes producidos dependen de las tasas de consumo de suelo, y que el 40 a $45 \%$ de tales volúmenes descansan en las demandas de agua. Por otro lado, el
$28 \%$ al $35 \%$ de los volúmenes producidos se traducen en residuos, esto quiere decir, que los rendimientos por unidad de volumen de materia prima principal respecto a producto terminado oscilan entre el $45 \%$ y $65 \%$. Esto quiere decir, que se requiere mejorar estas cadenas en términos de eficiencia y rendimiento. 


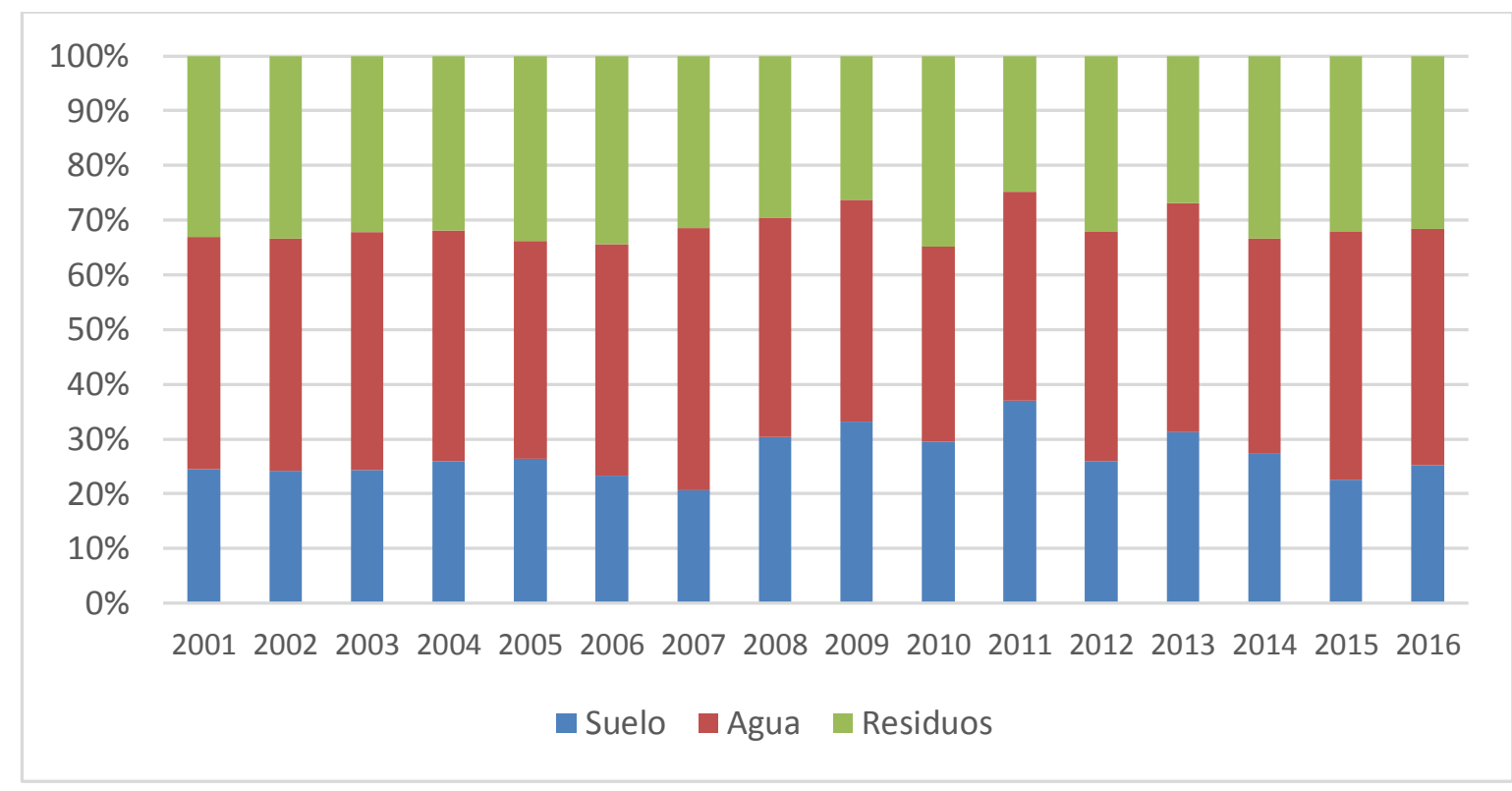

Figura 10. Composición de presiones y cargas ambientales en las cadenas agroindustriales de tipo $C 2$

Por último, para el caso de las cadenas agroindustriales del tipo C3, que se aprecian en la Figura 11, se tienen importantes tasas de consumo para

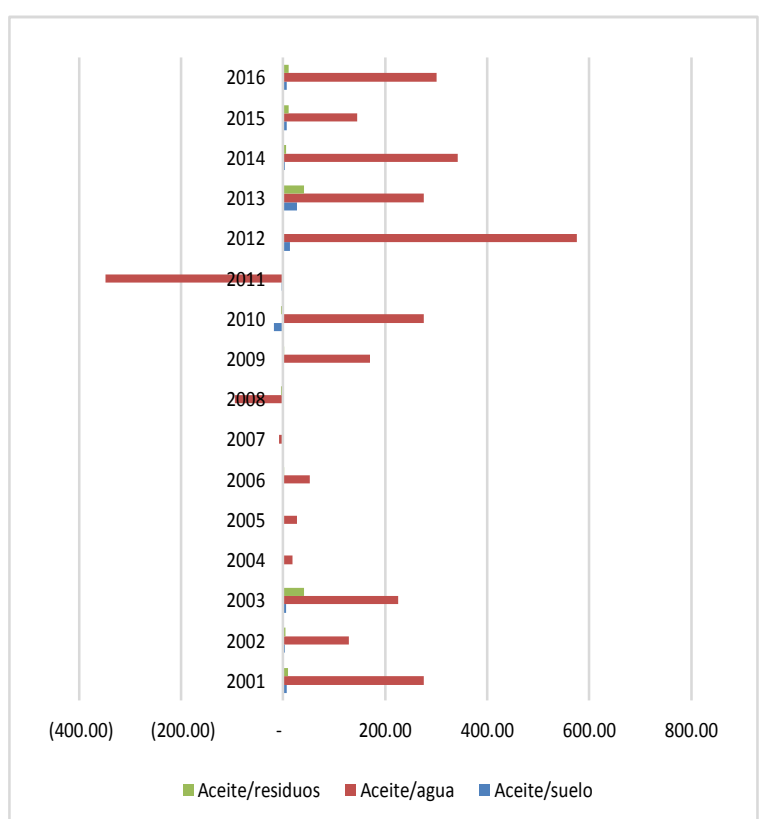

Fig. 11-A: Tasas de consumo de recursos naturales (suelo Fig. 11-B: Tasas de consumo de recursos naturales (suelo

\& agua) y de generación de residuos para C3 (caso aceite \& agua) y de generación de residuos para C3 (caso grasas). vegetal). los casos de aceites y grasas, y menores tasas en cadenas de derivados lácteos y embutidos.

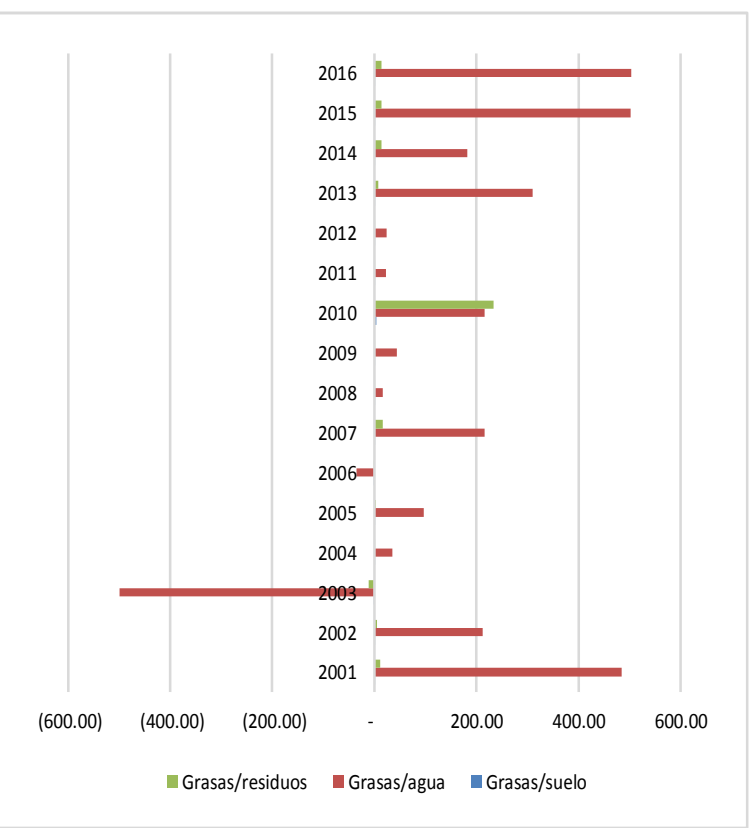




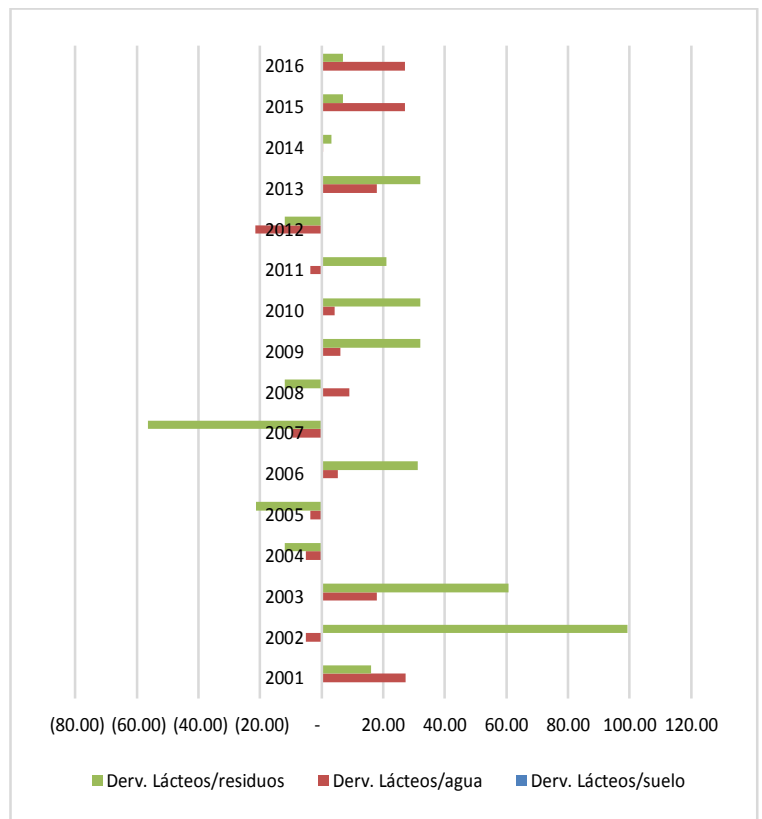

Fig. 11-C: Tasas de consumo de recursos naturales (suelo \& agua) y de generación de residuos para $\mathrm{C} 3$ (caso derivados lácteos).

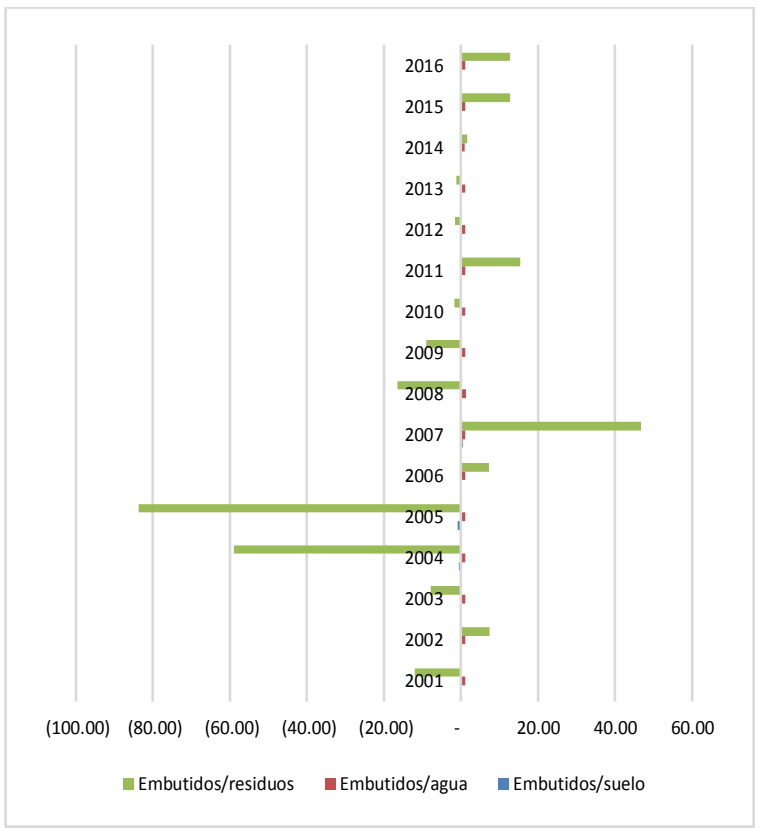

Fig. 11-D: Tasas de consumo de recursos naturales (suelo \& agua) y de generación de residuos para C3 (caso embutidos).

Figura 11. Tasas de consumo de recursos naturales y generación de residuos en C3

No se observan diferencias en cuanto a la relación entre volumen producido y presiones ambientales (suelo \& agua), así como para el caso de las cargas ambientales (residuos sólidos). De ese modo, se puede concluir, que a pesar de presentarse contracciones significativas en cadenas productivas particulares (derivados lácteos y embutidos), la naturaleza expansiva del proceso se mantiene, es decir, que todo aumento de la producción o decrecimiento se hace a expensas de mayor capital natural (recursos naturales) o mayor contaminación (residuos sólidos), tal como se aprecia de los resultados expuestos en la Tabla 4.

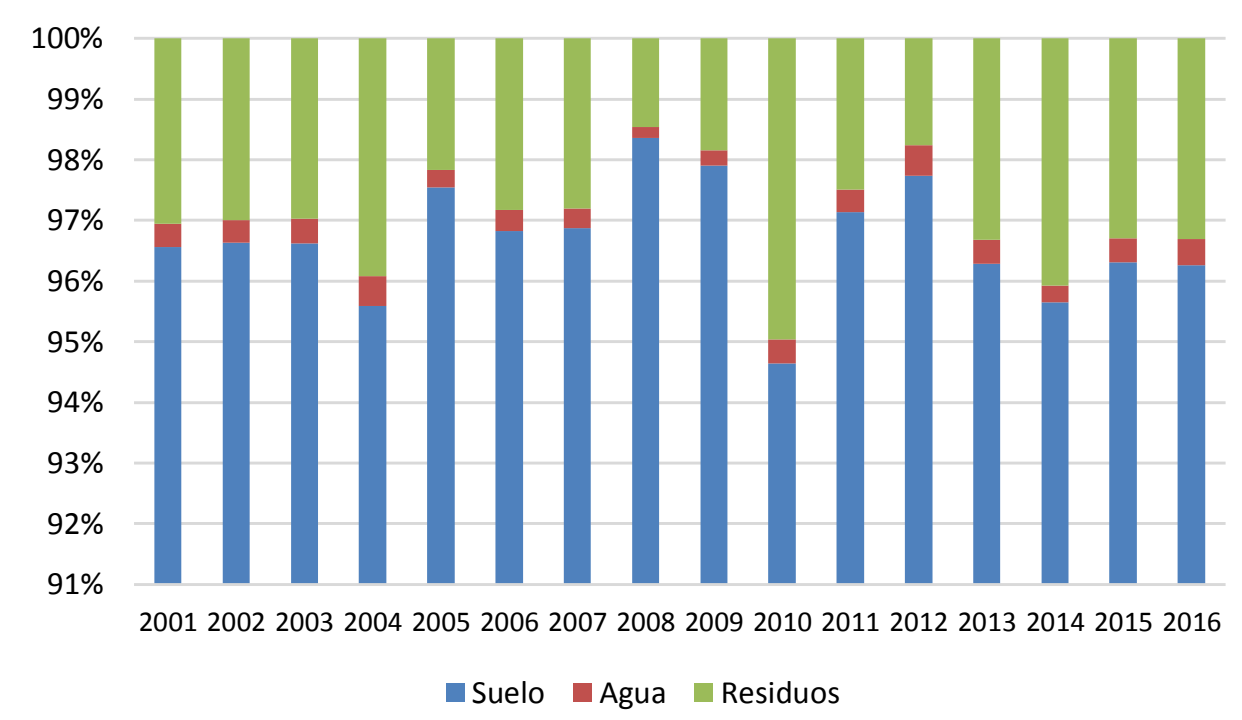

Figura 12. Composición de presiones y cargas ambientales en las cadenas agroindustriales de tipo C3 
Tabla 4

Producción, consumo de recursos naturales (capital natural) y generación de residuos para cadenas agroindustriales del tipo C3 (2000-2016)

\begin{tabular}{|c|c|c|c|c|c|c|c|c|c|c|c|c|c|c|c|c|}
\hline \multirow{2}{*}{ Años } & \multicolumn{4}{|c|}{ Producción en Tm } & \multicolumn{3}{|c|}{ Aceite } & \multicolumn{3}{|c|}{ Grasa } & \multicolumn{3}{|c|}{ Derv. Lácteos } & \multicolumn{3}{|c|}{ Embutidos } \\
\hline & Aceite & Grasa & $\begin{array}{l}\text { Derv. } \\
\text { Lácteos }\end{array}$ & $\begin{array}{l}\text { Embu- } \\
\text { tidos }\end{array}$ & $\begin{array}{l}\text { Suelo } \\
\text { ha* }^{*}\end{array}$ & $\begin{array}{l}\text { Agua } \\
\mathrm{m}^{3 *}\end{array}$ & $\begin{array}{c}\text { Residuos } \\
\mathrm{Tm}^{*}\end{array}$ & $\begin{array}{l}\text { Suelo } \\
\text { ha }^{*}\end{array}$ & $\begin{array}{l}\text { Agua } \\
\mathrm{m}^{3 *}\end{array}$ & $\begin{array}{l}\text { Residuos } \\
\mathrm{Tm}^{*}\end{array}$ & $\begin{array}{l}\text { Suelo } \\
\text { ha* }^{*}\end{array}$ & $\begin{array}{l}\text { Agua } \\
\mathrm{m}^{3 *}\end{array}$ & $\begin{array}{c}\text { Residuos } \\
\mathrm{Tm}^{*}\end{array}$ & $\begin{array}{l}\text { Suelo } \\
\text { ha }^{*}\end{array}$ & $\begin{array}{l}\text { Agua } \\
\mathrm{m}^{3 *}\end{array}$ & $\begin{array}{c}\text { Residuos } \\
\mathrm{Tm}^{*}\end{array}$ \\
\hline 2000 & 164,880 & 69,277 & 7,340 & 23,560 & 25,366 & 588 & 17,578 & 415,662 & 145 & 6,491 & 191,145 & 274 & 45 & 188,480 & 2,161 & 1,903 \\
\hline 2001 & 162,385 & 70,504 & 7,486 & 23,970 & 24,976 & 579 & 17,308 & 422,895 & 148 & 6,604 & 194,873 & 280 & 46 & 191,703 & 2,198 & 1,935 \\
\hline 2002 & 173,867 & 64,066 & 9,847 & 27,734 & 28,827 & 669 & 19,976 & 336,319 & 117 & 5,252 & 293,796 & 253 & 70 & 243,111 & 2,788 & 2,454 \\
\hline 2003 & 188,016 & 60,911 & 8,025 & 28,724 & 31,534 & 732 & 20,323 & 354,359 & 124 & 5,533 & 88,274 & 243 & 40 & 208,647 & 2,392 & 2,106 \\
\hline 2004 & 184,766 & 62,045 & 10,780 & 29,974 & 23,627 & 548 & 16,666 & 446,893 & 156 & 6,978 & 475,031 & 233 & 39 & 221,942 & 2,545 & 2,241 \\
\hline 2005 & 186,694 & 66,945 & 10,678 & 30,895 & 26,703 & 619 & 18,315 & 591,736 & 207 & 9,240 & 182,189 & 261 & 43 & 218,299 & 2,503 & 2,204 \\
\hline 2006 & 202,004 & 68,397 & 12,076 & 35,497 & 39,320 & 912 & 25,328 & 471,502 & 165 & 7,363 & 368,799 & 530 & 88 & 391,666 & 2,735 & 3,955 \\
\hline 2007 & 204,227 & 78,482 & 15,123 & 39,422 & 27,363 & 635 & 18,757 & 968,845 & 178 & 7,983 & 690,805 & 206 & 34 & 366,288 & 2,844 & 3,698 \\
\hline 2008 & 188,145 & 77,763 & 19,393 & 44,630 & 12,411 & 805 & 23,131 & 385,890 & 135 & 6,026 & 939,311 & 680 & 33 & 455,744 & 2,958 & 4,602 \\
\hline 2009 & 204,996 & 80,372 & 16,953 & 44,866 & 36,103 & 838 & 33,070 & 554,123 & 193 & 8,653 & 47,208 & 274 & 31 & 197,606 & 2,266 & 1,995 \\
\hline 2010 & 240,562 & 96,696 & 19,001 & 47,339 & 34,089 & 871 & 25,019 & 558,614 & 195 & 8,723 & 532,060 & 764 & 30 & 313,602 & 3,596 & 3,166 \\
\hline 2011 & 228,421 & 94,915 & 20,053 & 50,184 & 39,050 & 906 & 10,180 & 337,486 & 118 & 5,270 & 335,420 & 482 & 80 & 345,728 & 3,965 & 3,491 \\
\hline 2012 & 249,304 & 96,082 & 20,901 & 51,367 & 40,612 & 942 & 27,061 & 480,451 & 168 & 7,502 & 308,055 & 442 & 74 & 247,677 & 2,840 & 2,501 \\
\hline 2013 & 294,247 & 110,176 & 8,354 & 42,824 & 42,236 & 980 & 28,143 & 610,413 & 213 & 9,532 & 112,432 & 161 & 27 & 144,945 & 1,271 & 1,119 \\
\hline 2014 & 307,654 & 114,179 & 8,437 & 44,838 & 46,088 & 1,019 & 30,604 & 673,379 & 235 & 9,818 & 219,415 & 315 & 52 & 348,703 & 3,743 & 3,521 \\
\hline 2015 & 321,671 & 118,328 & 8,522 & 46,947 & 48,096 & 1,116 & 31,938 & 696,988 & 243 & 10,112 & 221,588 & 318 & 53 & 364,368 & 4,178 & 3,679 \\
\hline 2016 & 336,327 & 122,627 & 8,607 & 49,156 & 50,192 & 1,165 & 33,330 & 721,425 & 252 & 10,416 & 223,782 & 321 & 53 & 380,738 & 4,366 & 3,844 \\
\hline
\end{tabular}

* Valores estimados con base a balances de masa por producto según diagramas de flujo de procesos estandarizados por sub-sector.

Fuente: (Prom Perú, 2015) y estimaciones propia

En este clúster también se aprecian importantes presiones ambientales, así como cargas (Figura 12). Sin embargo, no debe soslayarse el hecho, que los resultados ofrecidos tienen un sesgo al considerar un grupo de productos agroindustriales que descansan en insumos agrícolas como pecuarios que ejercen importantes presiones sobre el suelo. En el caso del agua se puede reproducir una argumentación similar a la ofrecida para el caso de las cadenas productivas del tipo C2. Nótese, en este caso, que la generación de residuos sólidos, es también significativa y hace pensar en la baja eficiencia y menor rendimiento de tales cadenas.
Hacia un modelo interpretativo de la sostenibilidad ambiental en la agroindustria peruana

El modelo propuesto por Pearce y Turner (1995) es válido para interpretar la relación entre crecimiento productivo del sector agroindustrial en sus diferentes cadenas productivas y las tasas de consumo de los recursos naturales, así como de las cargas ambientales o en este caso, generación de residuos sólidos. Se aprecia que para el caso de las presiones ambientales (Figura 13), que las agroindustrias del tipo C3, para el periodo 2000-2016, se desarrolló básicamente por un modelo expansivo, es decir mayor 
volumen producido y mayor presiones ambientales (agua \& suelo) (Figuras 13-A y 13-B). Sin embargo, es menester anotar que existen periodos temporales en los cuales, se obtuvieron tasas negativas para el consumo de agua y suelo, lo cual indicaría de un proceso mucho más complejo y afectado básicamente por el efecto de la importación de insumos. Por su parte, para el caso de las cadenas agroindustriales del tipo $\mathrm{C} 2$, se tienen un modelo similar, con amplio predominio

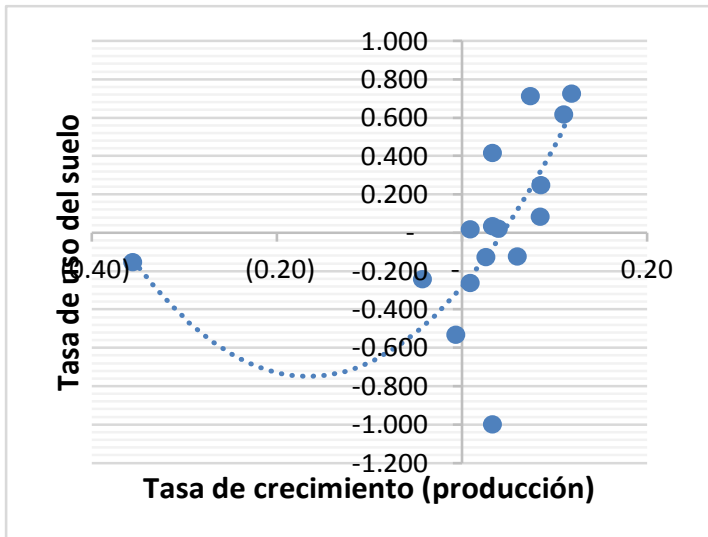

Fig. 13-A: Relación entre tasas de crecimiento (producción) y uso del suelo para cadenas agroindustriales tipo C3.

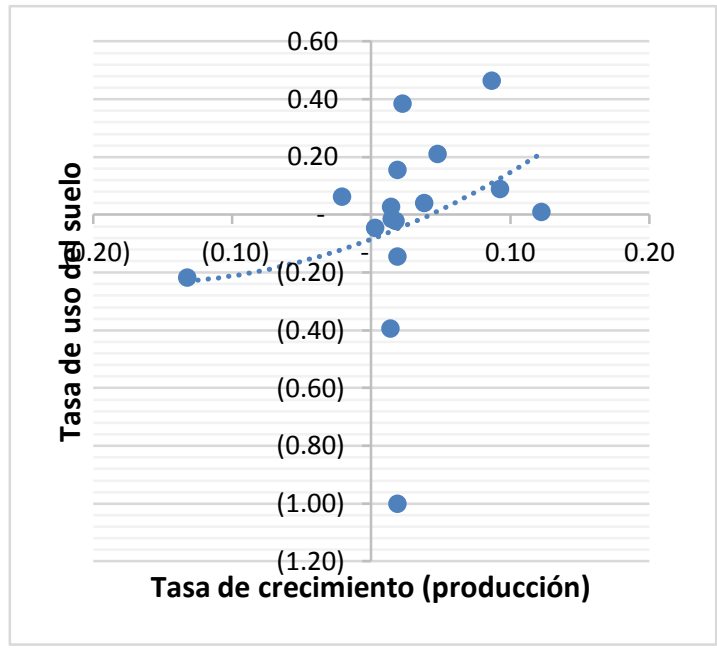

Fig. 13-C: Relación entre tasas de crecimiento (producción) y uso del suelo para cadenas agroindustriales tipo $\mathrm{C} 2$. de tasas crecientes de producción y tasas crecientes de uso del capital natural en términos de agua y suelo. En este caso también se presentan tasas negativas de consumo de recursos combinadas con tasas incrementales de producción de bienes del tipo C2. Esto alude nuevamente al efecto de la importación de insumos, muchas veces más baratos, de mejor calidad y en volúmenes importantes. Comentario que también es aplicable al caso de las cadenas $\mathrm{C} 1$.

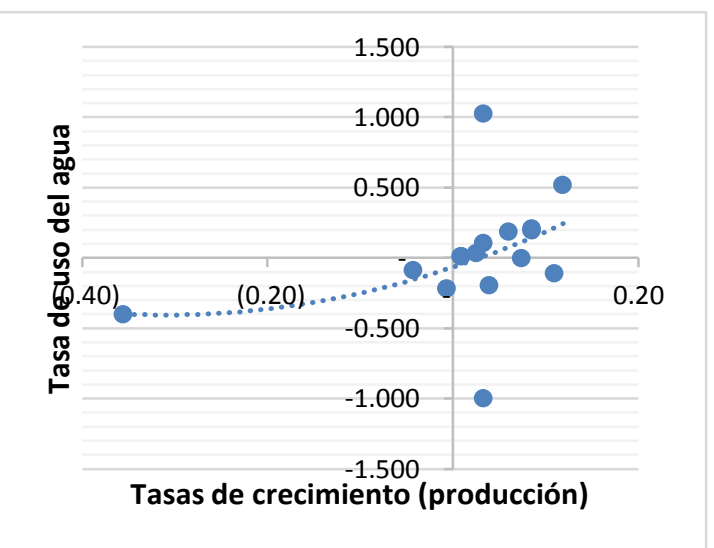

Fig. 13-B: Relación entre tasas de crecimiento (producción) y consumo de agua para cadenas agroindustriales tipo $\mathrm{C} 3$.

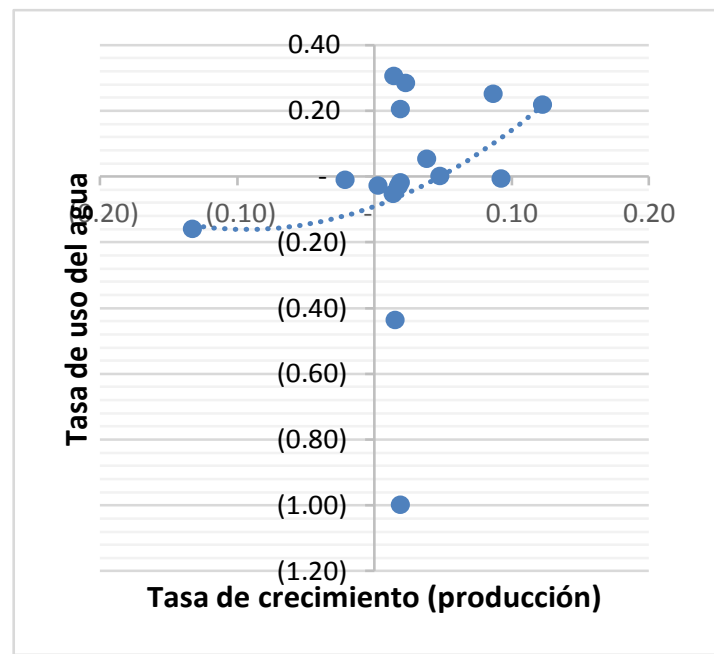

Fig. 13-D: Relación entre tasas de crecimiento (producción) y consumo de agua para cadenas agroindustriales tipo $\mathrm{C} 2$. 


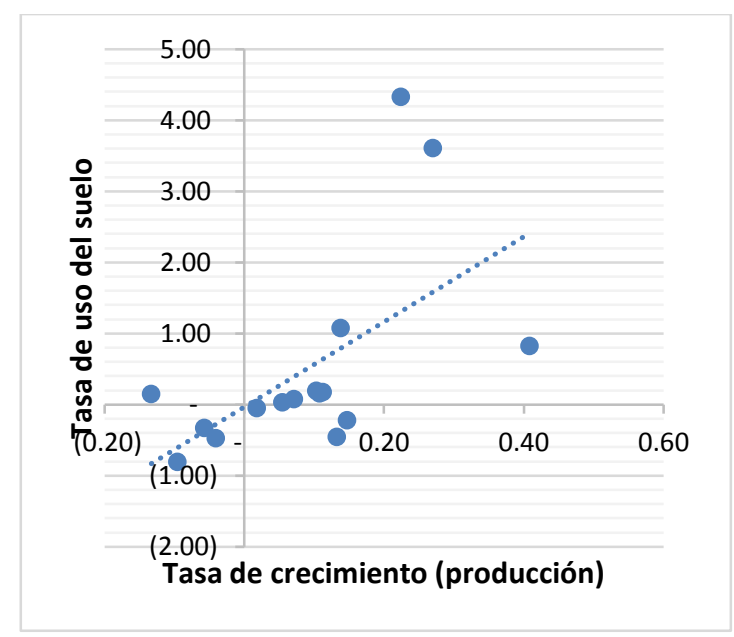

Fig. 13-E: Relación entre tasas de crecimiento (producción) y uso del suelo para cadenas agroindustriales tipo C1.

Figura 13. Presiones ambientales (suelo y agua) en términos de consumo respecto al volumen producido por cadenas agroindustriales para el periodo (2000-2016)

En el caso de las cargas ambientales, se aprecia un patrón similar, que alude a la vigencia del modelo Kuznets ambiental, según el cual, en la fase expansiva de la economía, todo incremento de la producción se hace a expensas del ambiente, es decir a un costo ambiental

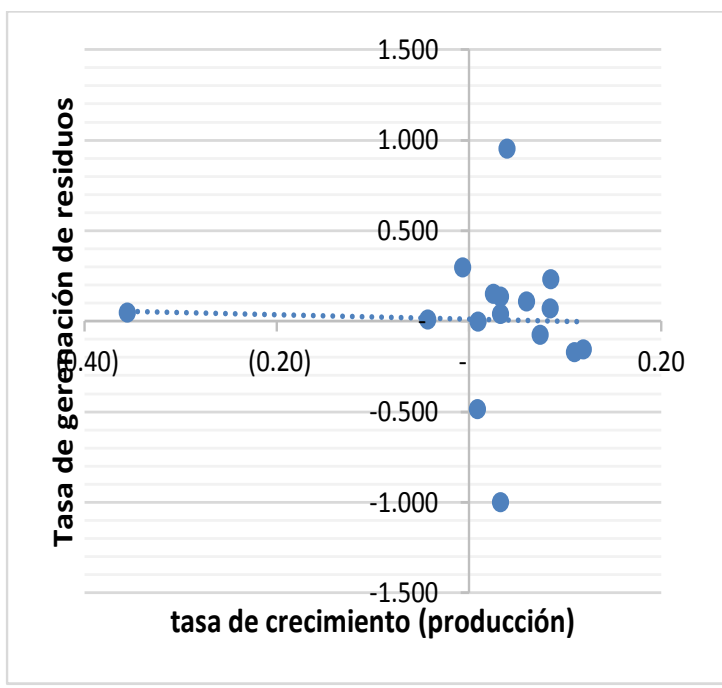

Fig. 14-A: Relación entre tasas de crecimiento (producción) y generación de residuos sólidos para cadenas agroindustriales tipo $\mathrm{C} 3$.

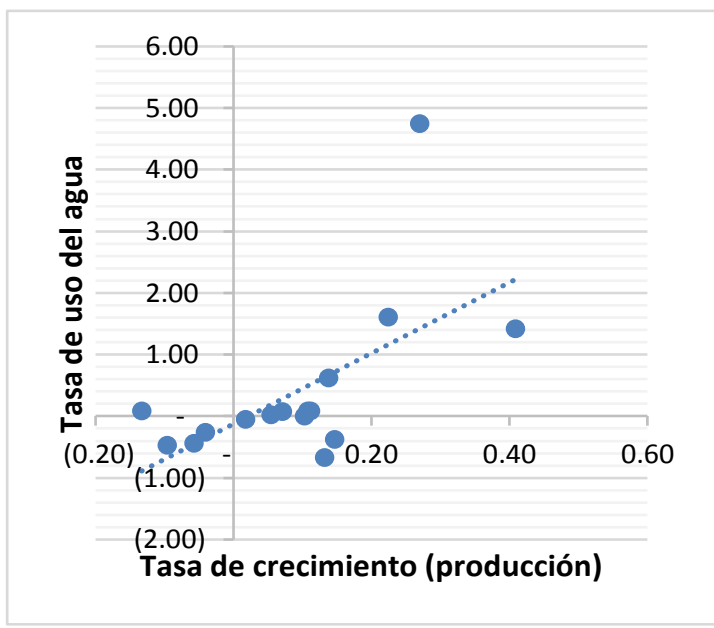

Fig. 13-F: Relación entre tasas de crecimiento (producción) y consumo de agua para cadenas agroindustriales

alto, derivado de la externalidad de contaminar más con cada aumento de la producción. Esta causalidad se aprecia para la mayoría de las cadenas agroindustriales, tanto del tipo $\mathrm{C} 1$ como las del tipo C2 y C3 (Figura 14).

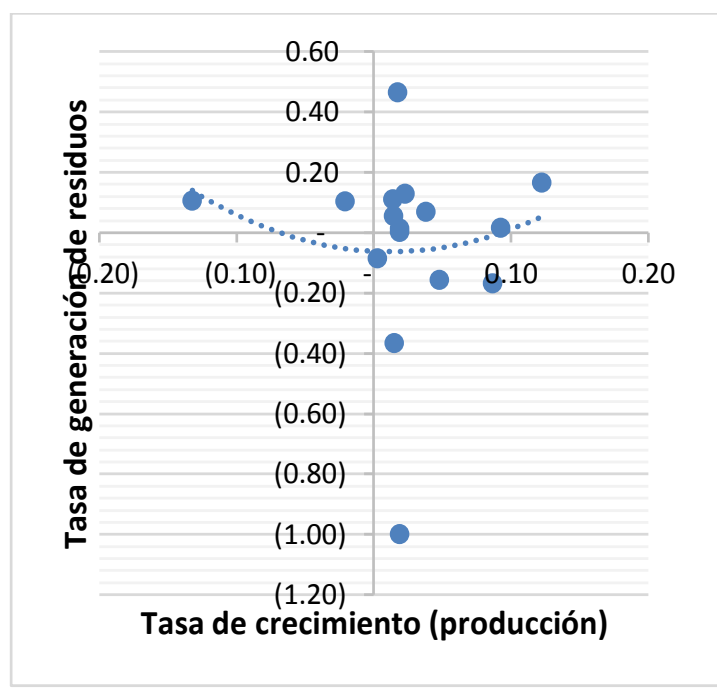

Fig. 14-B: Relación entre tasas de crecimiento (producción) y generación de residuos sólidos para cadenas agroindustriales tipo $\mathrm{C} 2$. 


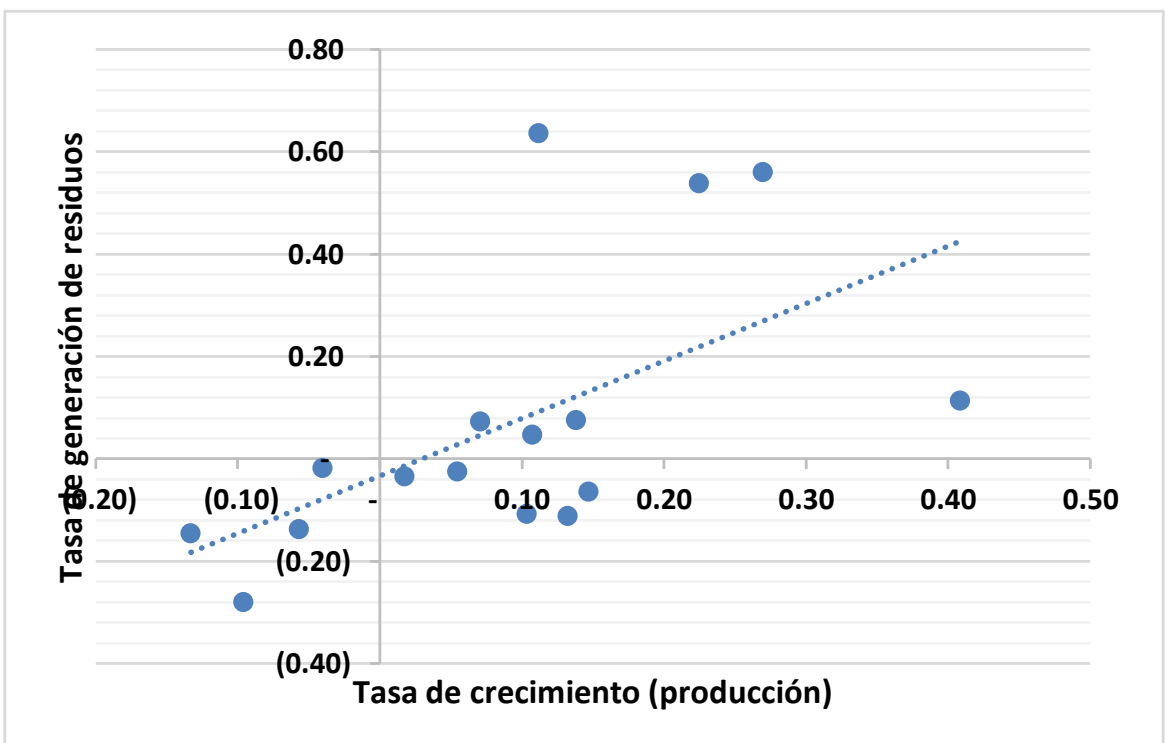

Fig. 14-C: Relación entre tasas de crecimiento (producción) y generación de residuos sólidos para cadenas agroindustriales tipo $\mathrm{C} 1$.

Figura 14. Cargas ambientales (generación de residuos sólidos) según cadenas agroindustriales para el periodo 2000-2016

\section{Discusión}

El modelo propuesto por Pearce y Turner (1995) es válido para interpretar la relación entre crecimiento productivo del sector agroindustrial en sus diferentes cadenas productivas y la tasas de consumo de los recursos naturales, así como de la cargas ambientales o en este caso, generación de residuos sólidos. En ese contexto, se han propuestos diversos indicadores para medir la sostenibilidad ambiental de los procesos económicos y sociales, tales como la huella ecológica (Suárez, Estrada, \& Spoor, 2017), que compara los niveles de consumo actuales con los consumos posibles de sostener. Se sostiene, por ejemplo, que las economías con mayores tasas de crecimiento tienen mayor huella ecológica y por tanto son menos sostenibles, lo cual es más que evidente en el caso de las ciudades (Muñiz, y otros, 2016; Defilippi, 2012).
Algunos consideran medir la cantidad de habitad silvestres que se conserva en términos de bio-diversidad (McRae, Deinet, \& Freeman, 2017). En cambio, otros autores consideran que la sostenibilidad depende no solo de las tasas de crecimiento ni del quantum de "capital natural" que se emplea para lograrlas, sino que está vinculado con el uso del capital humano y de las tasas de reposición del mismo(Burbano, 2005; González, Montes, Santos, \& Monedero, 2008; Gómez-Baggethum \& de Groot, 2007). En el caso que se expone, la relación entre tasas es positiva, y que tal condición se mantiene en el mediano largo plazo. Por tanto, el análisis de sostenibilidad global que se propone, es consistente con las consideraciones teóricas de GuhlNannetti(2017) o las señaladas por Hinterberger, Luks\& Schmidt-Bleek (1997). De ese modo, se concluye que la agroindustria nacional en el periodo analizado (2000-2016) describe una 
trayectoria expansiva, según la cual y siguiendo el modelo teórico de Pearce \& Turner (1995) se requiere por cada unidad de incremento de producción una unidad equivalente de recursos naturales. Situación última que conlleva a suponer que, en el largo plazo, la agroindustria en sus diferentes clústeres sea insostenible, dada las enormes presiones ambientales (suelo \& agua).

Por el lado de las cargas ambientales, las trayectorias que describen las agroindustrias del tipo C1, C2 y C3 corresponden a la fase expansiva del crecimiento del modelo teórico de Kuznets, según el cual, cualquier aumento de la economía o la producción se realiza a expensas del ambiental, aumentado la externalidad derivada de la contaminación, en este caso, de la generación de residuos sólidos. Parte del problema expansivo de la producción agroindustrial en el Perú se explica por los bajos niveles de eficiencia técnica y en particular por los bajos rendimientos, en términos de materia prima respecto al producto terminado. De forma tal que el promedio del rendimiento difiere según el tipo de cadena agroindustrial y está en el orden del 45 al $52 \%$.

\section{Referencias}

Arteaga, W. (s/f). El sector agro y agroindustria. Oportunidades comerciales y tendencias de mercado. LIma: Promperú.

Bovenberg, A., \& Smulders, S. (1995). Environmentantal Quality and Pollution-Argumenting Technological Change in a Two-Section Endogenous Growth Model. Journal of Public Economics, 369-391.

Burbano, H. (2005). La crisis ambiental del mundo al iniciar el siglo XXi: Elementos para su análisis. Tendencias Revista de la Facultad de Ciencias Económicas y Administrativas. Universidad de Nariño, 21-25.

Chiang, A., \& Wainwrigth, K. (2006). Métodos fundamentales de Economía Matemática. México: McGraw Hill.
Chiesura, A. d. (2003). Critical natural capital: a socio-cultural perspective. Ecologycal Economics (44), 219-231.

Chiesura, A., \& de Groot, R. (2003). Critical natural capital: a sociocultural perspective. Ecologycal Economics(44), 219-231.

Costanza R, J., Cumberland, H., Daly, R., Goodland, \& Norgaard., R. (1999). Una introducción a la Economía Ecológica. México: Compañía Editorial Continental. S.A. de C.V.

Dasgupta, P. (1993). Natural resources in an Age of Substitutability. En A. y. Kneese, Handboock of Natural Resources And Energy Economics. North-Holland: Elsevier.

De Groot., R. S. (1992). Functions of Nature: Evaluation of Nature in Environmental Planning, 
Management and Decision Making. Groningen: Wolters Noordhoff.

Defilippi, T. (2012). Consumo Ambiental y sostenibilidad urbana: caso de Lima y Lurin-Pachacamac. Tesis para optar el grado de Maestra. Lima: UNFV.

Ekins, P., Simon, S., Deutsch, L., \& Folke, C. y. (2003). A Framework for the practical application of the concepts of critical natural capital and strong sustainability. Ecological Economics(44 (2-3)), 165-185.

Escobal, J. \&. (2002). Un sistema de indicadores lideres del nivel de actividad para la economía peruana. Lima: Grade.

Escot, L., \& Galindo, M. (1999). Medio ambiente y crecimiento económico. En M. A. Galindo, Economía y medio ambiente (págs. 45-78). Madrid: Bilbioteca Nueva.

Gómez-Baggethum, E., \& de Groot, R. (2007). Capital natural y funciones de los ecosistemas: explorando las bases ecológicas de la economía. ecosistemas. Revista ciientifica y técnica de ecología y medio ambiente, 4-14.

González, J., Montes, C., Santos, I., \& Monedero, C. (2008). Inviertiendo en capital natural: un marco para integrar la sostenibilidad ambiental en las políticas de cooperación. ecosistemas. Revista cientifica de ecología y medio ambiente, 52-69.
Guhl-Nannetti, E. (10 de febrero de 2017). La sostenibilidad $y$ los páramos. Obtenido de quinaxi: http://www.quinaxi. $\mathrm{org} /$ index.php?option $=\mathrm{com}_{-}$ z o o $\&$ task = it e m \& it e m $\mathrm{id}=153 \&$ Itemid $=147$

Gujarati, D. (2006). Principios de econometría. Madrid: McGraw Hill.

Hanley, N., \& Atkinson, G. (2003). Economics and sustainable development: what have we learnt, and what do we still need to learn? En M. L. In F. Berkhout, Negotiating Environmental Change: New Perspectives from Social Science. Cheltenham, UK: Edwar Elgar.

Hinterberger, F., Luks, F., \& SchmidtBleek, F. (1997). Material flows vs.natural capital': What makes an economy sustainable? Ecological economics, 1-14.

McRae, L., Deinet, S., \& Freeman, R. (2017). The Diversity-Weighted Living Planet Index: Controlling for Taxonomic Bias in a Global Biodiversity Indicator. PLoS one. e0169156. doi:10.1371/journal. pone.0169156.

Michel, P., \& Rotillon, G. (1992). Pollution's disutility and endogenous growth. Paris: Mimeo.

Muñiz, I., Rojas, C., Busuldu, C., García, A., Filipe, M., \& Quintana, M. (2016). Forma urbana y Huella Ecológica en el Área Metropolitana de Concepción 
(Chile). Revista EURE (Santiago), 209-330.

Pearce, D., \& Turner, R. K. (1995). Economía de los recursos naturales $y$ del medio ambiente. Madrid: Celeste Ediciones.

Porter, M. E. (2015). Ventaja competitiva: creación y sostenimiento de un desempeño superior. . México: Grupo Editorial Patria.

Porter, M. E., \& Heppelmann, J. E. (2016). How smart, connected products are transforming competition. Harvard business review, 92(11), 18-24.

Prom Perú. (2015). Informe anual. Desembolvimiento agroexportador del Perú. Lima: Prom Perú.

Schumacher, E. (1973). Lo pequeño es hermoso. Madrid: Ediciones Orbis.

Schumpeter, J. (1934). The theory of Economic Development. Cambridge, Massachusetts: Harvard University Press.
SIEE. (1992). Investign in natural capital: The ecological economics approach to sustainability. Natural Capital. Reunión Bianual. Sociedad Internacional de Economía Ecológica.

Suárez, C. R., Estrada, C. M., \& Spoor, C. M. (2017). El índice desempeño ambiental y la resiliencia social en los ecosistemas. . Universidad y Sociedad, 6-12.

Tejada, T. (2008). El Cultivo de Trigo en la Sierra Norte del Perú. (Triticum aestivum L. y Triticum durum L.). Lima: INIA.

Veliz, C. (2009). Técnicas de predicción: Regresión y series de tiempo. Lima: PUCP.

Wackernagel, M., \& Rees, W. (1997). Perceptual and structural barriers to investing in natural capital: Economics from an ecological footprint perspective. Ecological Economics $N^{\circ} 20$. 


\section{Apéndice I}

Para el caso de arroz pilado (C1)

\begin{tabular}{ccc}
\hline Ítems & Arroz pilado \\
\hline & $\begin{array}{c}\text { Mat. Prima 1 } \\
\text { Arroz con cáscara }\end{array}$ & 85.0 \\
\hline Agua & Vapor de agua & 5.4 \\
Merma & Impureza & 1.6 \\
Otros 1 & Pajilla & 17.2 \\
Otros 2 & Tierra y residuos & 0.61 \\
Producto & Arroz blanco & 52.4 \\
Sub. Prod. 1 & Nelen y granos partidos & 0.5 \\
Sub. Prod. 2 & Polvillo & 7.0 \\
Sub. Prod. 3 & Arroz quebrado & 0.5 \\
Total inputs & & 85.0 \\
Total outputs & & 85.0 \\
\hline
\end{tabular}

Para el caso de productos C3

\begin{tabular}{|c|c|c|c|c|}
\hline Ítems & Aceite & & Embutido & \\
\hline Mat. Prima 1 & Nuez (kg) & 0.7 & Carne de res y cerdo $(\mathrm{kg})$ & 760 \\
\hline Mat. Prima 2 & & & Hielo $(\mathrm{kg})$ & 45 \\
\hline Mat. Prima 3 & & & Texturizado de soya $(\mathrm{kg})$ & 50 \\
\hline Mat. Prima 4 & & & Tocino $(\mathrm{kg})$ & 30 \\
\hline Mat. Prima 5 & & & Especias $(\mathrm{kg})$ & 34 \\
\hline Mat. Prima 6 & & & Aditivos (kg) & 36.9 \\
\hline Mat. Prima 7 & & & Hielo (kg) & 45 \\
\hline Mat. Prima 8 & & & Omega $3(\mathrm{~kg})$ & 70 \\
\hline Agua & & & & 90 \\
\hline Merma & Impureza $(\mathrm{kg})$ & 0.00 & Molido (kg) & 4.59 \\
\hline Otros 1 & Cáscara (kg) & 0.10 & Mezclado (kg) & 4.79 \\
\hline Otros 2 & Almendras dañadas $(\mathrm{kg})$ & 0.02 & Embutido (kg) & 22.71 \\
\hline Otros 3 & Perdidas $(\mathrm{kg})$ & 0.01 & Ahumado (kg) & 42.17 \\
\hline Otros 4 & Perdidas (kg) & 0.18 & Reposo (kg) & 4.98 \\
\hline Otros 5 & Almendra + Hexano $(\mathrm{kg})$ & 0.17 & & \\
\hline Otros 6 & Hexano (lt) & 5.57 & & \\
\hline Otros 7 & Aceite virgen $(\mathrm{kg})$ & 0.27 & Chorizo parrilla (kg) & 991.66 \\
\hline Total inputs & & 0.7 & & 1070.9 \\
\hline Total outputs & & 0.7 & & 1070.9 \\
\hline
\end{tabular}




\section{Para el caso de productos C2}

\begin{tabular}{|c|c|c|c|c|c|c|c|c|c|c|}
\hline Ítems & \multicolumn{2}{|c|}{ Alimento balanceado } & \multicolumn{2}{|l|}{ Fideos } & \multicolumn{2}{|c|}{ Harina } & \multicolumn{2}{|c|}{ Avena } & \multicolumn{2}{|c|}{ Sémola } \\
\hline Mat. Prima 1 & $\begin{array}{l}\text { Suplemento proteico } \\
(\mathrm{kg})\end{array}$ & & $\begin{array}{l}\text { Harina de } \\
\text { trigo }(\mathrm{kg})\end{array}$ & 20 & Ocara (g) & 1068.8 & $\begin{array}{c}\text { Harina de } \\
\text { quinua } \\
(\mathrm{kg})\end{array}$ & 1.5 & $\begin{array}{l}\text { Sémola de } \\
\text { trigo (gr) }\end{array}$ & 708.36 \\
\hline Mat. Prima 2 & Elemento base (kg) & 650.64 & $\begin{array}{l}\text { Harina de soya } \\
\qquad(\mathrm{kg})\end{array}$ & 4.6 & & & $\begin{array}{l}\text { Harina de } \\
\text { soya }(\mathrm{kg})\end{array}$ & 0.8 & $\begin{array}{l}\text { Harina papa } \\
\text { criolla (gr) }\end{array}$ & 86.64 \\
\hline Mat. Prima 3 & Premezclas (kg) & & $\begin{array}{c}\text { Proteína } \\
\text { concentrada de } \\
\text { soya }(\mathrm{kg})\end{array}$ & 1.6 & & & Agua $(\mathrm{kg})$ & 1.3 & Huevo (gr) & 190.08 \\
\hline Mat. Prima 4 & Suero (kg) & 195 & $\begin{array}{c}\text { Proteína } \\
\text { aislada de soya } \\
\quad(\mathrm{kg})\end{array}$ & 1.6 & & & $\begin{array}{c}\text { Hojuelas } \\
\text { de avena } \\
(\mathrm{kg})\end{array}$ & 1.6 & Aceite (gr) & 18.48 \\
\hline Mat. Prima 5 & & & $\begin{array}{c}\text { Harina de } \\
\text { zanahoria (kg) }\end{array}$ & 0.36 & & & $\begin{array}{l}\text { Espirulina } \\
(\mathrm{kg})\end{array}$ & 0.15 & Sal (gr) & 6.36 \\
\hline Mat. Prima 6 & & & $\begin{array}{c}\text { Harina de } \\
\text { espinaca }(\mathrm{kg})\end{array}$ & 0.36 & & & $\begin{array}{c}\text { Miel de } \\
\text { abeja }(\mathrm{kg})\end{array}$ & 1 & Agua (gr) & 190.08 \\
\hline Mat. Prima 7 & & & Huevo (kg) & 2.86 & & & $\begin{array}{c}\text { Azúcar } \\
(\mathrm{kg})\end{array}$ & 1.5 & & \\
\hline Mat. Prima 8 & & & Sal (kg) & 0.352 & & & $\begin{array}{c}\text { Esencia } \\
\text { de vainilla } \\
(\mathrm{kg})\end{array}$ & 0.05 & & \\
\hline Mat. Prima 9 & & & $\begin{array}{c}\text { Aceite de oliva } \\
\text { (lt) }\end{array}$ & 0.352 & & & & & & \\
\hline Agua & Agua $(\mathrm{kg})$ & 195.06 & Agua (lt) & 8.8 & Agua (g) & 416.96 & & & & \\
\hline Merma & Recepción (kg) & 0.065 & Impurezas $(\mathrm{kg})$ & 19.264 & $\begin{array}{l}\text { Leche de } \\
\text { soya (g) }\end{array}$ & 509.39 & & & $\begin{array}{c}\text { Mezclado } \\
\text { ya amasado } \\
\text { (gr) }\end{array}$ & 80 \\
\hline Otros 1 & Molienda (kg) & 0.065 & & & $\begin{array}{l}\text { Impureza } \\
\text { (g) }\end{array}$ & 12.8 & & & $\begin{array}{l}\text { Extruccion } \\
\text { y moldeo } \\
\text { (gr) }\end{array}$ & 180 \\
\hline Otros 2 & Mezclado (kg) & 0.084 & & & & & & & Secado (gr) & 160 \\
\hline Otros 3 & Extrucción (kg) & 0.084 & & & & & & & & \\
\hline Otros 4 & Enfriamiento(kg) & 0.084 & & & & & & & & \\
\hline Otros 5 & Almacenamiento (kg) & 0.065 & & & & & & & & \\
\hline Otros 6 & Pesaje (kg) & 0.065 & & & & & & & & \\
\hline Otros 7 & Empaque(kg) & 0.065 & & & & & & & & \\
\hline Producto & $\begin{array}{c}\text { Concentrado para } \\
\text { cuy }(\mathrm{kg})\end{array}$ & 650 & Fideos (kg) & 21.62 & $\begin{array}{l}\text { Harina de } \\
\text { Ocara (g) }\end{array}$ & 129.65 & $\begin{array}{c}\text { Galleta } \\
\text { energética } \\
(\mathrm{kg})\end{array}$ & 7.9 & $\begin{array}{c}\text { Pasta } \\
\text { alimenticia }\end{array}$ & 780 \\
\hline Total inputs & & 845.64 & & 40.884 & & 1068.8 & & 7.9 & & 1200 \\
\hline Total outputs & & 845.64 & & 40.884 & & 1068.8 & & 7.9 & & 1200 \\
\hline
\end{tabular}


